\title{
THE VMC SURVEY. XIX. CLASSICAL CEPHEIDS IN THE SMALL MAGELLANIC CLOUD
}

\author{
V. Ripepi ${ }^{1}$, M. Marconi ${ }^{1}$, M. I. Moretti ${ }^{2}$, G. Clementini ${ }^{3}$, M.-R. L. Cioni ${ }^{4,5,6}$, R. De Griss ${ }^{7,8}$, J. P. Emerson ${ }^{9}$, \\ M. A. T. Groenewegen ${ }^{10}$, V. D. Ivanov ${ }^{11}$, and A. E. Piatti ${ }^{12,13}$ \\ ${ }^{1}$ INAF-Osservatorio Astronomico di Capodimonte, via Moiariello 16, I-80131, Naples, Italy; ripepi@oacn.inaf.it \\ ${ }_{2}^{2}$ IAASARS, National Observatory of Athens, 15236 Penteli, Greece \\ ${ }^{3}$ INAF-Osservatorio Astronomico di Bologna, via Ranzani, I-40127, Bologna, Italy \\ ${ }^{4}$ Universität Potsdam, Institut für Physik und Astronomie, Karl-Liebknecht-Strasse 24/25, D-14476 Potsdam, Germany \\ ${ }^{5}$ Leibniz-Institut für Astrophysik Potsdam, An der Sternwarte 16, D-14482 Potsdam Germany \\ ${ }^{6}$ Centre for Astrophysics Research, School of Physics, Astronomy and Mathematics, University of Hertfordshire, College Lane, Hatfield AL10 9AB, UK \\ ${ }^{7}$ Kavli Institute for Astronomy \& Astrophysics and Department of Astronomy, Peking University, Yi He Yuan Lu 5, Hai Dian District, Beijing 100871, China \\ ${ }^{8}$ International Space Science Institute-Beijing, 1 Nanertiao, Zhongguancun, Hai Dian District, Beijing 100190, China \\ ${ }^{9}$ School of Physics \& Astronomy, Queen Mary University of London, Mile End Road, London E1 4NS, UK \\ ${ }^{10}$ Koninklijke Sterrenwacht van België, Ringlaan 3, B-1180, Brussel, Belgium \\ ${ }^{11}$ European Southern Observatory, Karl-Schwarzschild-Strasse 2, D-85748 Garching bei München, Germany \\ ${ }^{12}$ Observatorio Astronómico, Universidad Nacional de Córdoba, Laprida 854, 5000, Córdoba, Argentina \\ ${ }^{13}$ Consejo Nacional de Investigaciones Científicas y Técnicas, Av. Rivadavia 1917, C1033AAJ, Buenos Aires, Argentina \\ Received 2015 December 24; accepted 2016 February 24; published 2016 June 1
}

\begin{abstract}
The "VISTA near-infrared $Y J K_{\mathrm{s}}$ survey of the Magellanic Clouds System" (VMC) is collecting deep $K_{\mathrm{s}}$-band time-series photometry of pulsating variable stars hosted by the two Magellanic Clouds and their connecting Bridge. In this paper, we present $Y, J, K_{\mathrm{s}}$ light curves for a sample of 4172 Small Magellanic Cloud (SMC) Classical Cepheids (CCs). These data, complemented with literature $V$ values, allowed us to construct a variety of period-luminosity (PL), period-luminosity-color (PLC), and period-Wesenheit (PW) relationships, which are valid for Fundamental (F), First Overtone (FO), and Second Overtone (SO) pulsators. The relations involving the $V, J, K_{\mathrm{s}}$ bands are in agreement with their counterparts in the literature. As for the $Y$ band, to our knowledge, we present the first CC PL, PW, and PLC relations ever derived using this filter. We also present the first near-infrared PL, PW, and PLC relations for SO pulsators to date. We used $\mathrm{PW}\left(V, K_{\mathrm{S}}\right)$ to estimate the relative SMC-LMC distance and, in turn, the absolute distance to the SMC. For the former quantity, we find a value of $\Delta \mu=0.55 \pm 0.04 \mathrm{mag}$, which is in rather good agreement with other evaluations based on CCs, but significantly larger than the results obtained from older population II distance indicators. This discrepancy might be due to the different geometric distributions of young and old tracers in both Clouds. As for the absolute distance to the SMC, our best estimates are $\mu_{\mathrm{SMC}}=19.01 \pm 0.05 \mathrm{mag}$ and $\mu_{\mathrm{SMC}}=19.04 \pm 0.06 \mathrm{mag}$, based on two distance measurements to the LMC which rely on accurate CC and eclipsing Cepheid binary data, respectively.
\end{abstract}

Key words: distance scale - Magellanic Clouds - stars: oscillations - stars: variables: Cepheids

Supporting material: figure set, machine-readable tables

\section{INTRODUCTION}

The Magellanic Clouds (MCs) are fundamental touchstones in the context of stellar populations and galactic evolution studies (see, e.g., Harris \& Zaritsky 2004, 2009; Ripepi et al. 2014a). Indeed, they are relatively close $\left(D_{\odot} \sim 50-60 \mathrm{kpc}\right.$ Westerlund 1997; Udalski et al. 1999), they are rich in stars of different ages, and their morphologies have been significantly affected by their dynamical interaction. In effect, there are clear signatures that the Small Magellanic Cloud (SMC), a gas-rich, dwarf irregular galaxy, is interacting with its neighbors, the Large Magellanic Cloud (LMC) and the Milky Way (MW). In particular, the MCs are connected by a Bridge dominated by $\mathrm{H}_{\mathrm{I}}$ gas but which also contains a significant stellar content (e.g., Irwin et al. 1985; Harris 2007). Like the Magellanic Stream, the Bridge may be the signature of the MCs' mutual gravitational effects and/or the impact of the MW (e.g., Putman et al. 1998; Hammer et al. 2015). In addition, the SMC Wing, the part of the SMC main body extending asymmetrically toward the LMC (Shapley 1940), could be the result of tidal interaction(s). Moreover, the bar of the SMC, traced by the galaxy's young populations, appears to be highly asymmetric and elongated, with its northeastern portion closer to us than its southwestern part (e.g., Welch et al. 1987; Haschke et al. 2012; Rubele et al. 2015; Scowcroft et al. 2016). In general, the morphology of the SMC appears to depend on the age of the stellar population used as a probe (see, e.g., Cioni et al. 2000a; Zaritsky et al. 2000; Dobbie et al. 2014; Deb et al. 2015, and references therein). The study of the structure of the SMC is further complicated by the presence of a considerable line-ofsight depth variation in the galaxy. Despite several studies, it appears that the precise extent of the line-of-sight depth and the three-dimensional (3D) geometry of the SMC are still rather uncertain (see, e.g., de Grijs et al. 2014, for a review). In fact, a comparison of the results in the recent literature adopting different methods, namely, horizontal-branch stars, RR Lyrae and/or Classical Cepheid (CC) variables, red-clump stars, full star formation-history (SFH) reconstruction, star clusters, etc., showed good qualitative agreement, but significant discrepancies in the quantitive description of the geometry of the SMC remain (see, e.g., Hatzidimitriou \& Hawkins 1989; Stanimirović et al. 2004; Glatt et al. 2008; Nidever et al. 2013; Deb et al. 2015; Rubele et al. 2015; Subramanian \& Subramaniam 2015 and references therein).

$\mathrm{CC}$ variables are at the base of the absolute calibration of the extragalactic distance scale (see, e.g., Freedman et al. 2001; 
Marconi et al. 2005; Riess et al. 2011; Fiorentino et al. 2013, and references therein) through their well known PeriodLuminosity (PL), Period-Luminosity-Color (PLC), and Period-Wesenheit (PW) relationships.

The CC PL relations have been demonstrated, by several authors, to show a nonnegligible dependence on both metallicity (see, e.g., Caputo et al. 2000; Romaniello et al. 2008; Bono et al. 2010, and references therein) and helium content (see Fiorentino et al. 2002; Marconi et al. 2005; Carini et al. 2014), and to exhibit a nonlinear behavior toward the longest periods (see, e.g., Caputo et al. 2000; Ngeow et al. 2008; Marconi 2009, and references therein). Both effects, combined with the intrinsic dispersion due to the finite width of the instability strip, are significantly reduced at near-infrared (NIR) wavelengths (Bono et al. 1999; Caputo et al. 2000; Marconi et al. 2005, 2010).

The PLC relations hold for each individual pulsator, since they result from the combination of the period-density, the Stefan-Boltzmann, and the Mass-Luminosity relations (see, e.g., Bono et al. 1999, for details), but they are affected by reddening and metallicity uncertainties. On the other hand, the PW relations are reddening-free by definition (e.g., Madore 1982; Caputo et al. 2000) and include a color term that accounts at least in part for the finite width of the instability strip. Moreover, they are less dependent on chemical composition than the PL relations. Furthermore, pulsation amplitudes are much smaller in the NIR than in the optical bands, and thus accurate mean magnitudes can be derived from a smaller number of phase points along the pulsation cycle with respect to the optical bands.

The "VISTA" near-infrared $Y J K_{\mathrm{s}}$ survey of the Magellanic Clouds System" (VMC; Cioni et al. 2011) aims at obtaining deep NIR photometric data in the $Y, J$, and $K_{\mathrm{s}}$ filters over a wide area covering the entire Magellanic system. VMC is a European Southern Observatory (ESO) public survey that is carried out with VIRCAM (VISTA InfraRed Camera; Dalton et al. 2006) on the ESO/VISTA telescope (Emerson et al. 2006). The main goals of the survey are to reconstruct the SFH and its spatial variation, as well as infer an accurate 3D map of the entire Magellanic system. The properties of pulsating stars observed by the VMC in the LMC and used as tracers of three different stellar populations, namely, CCs (younger than a few hundred Myr), RR Lyrae and Type II Cepheid stars (older than 9-10 Gyr), and Anomalous Cepheids (traditionally associated with an intermediate-age population of a few Gyr), ${ }^{15}$ have been discussed in recent papers by our team (Ripepi et al. 2012a, 2012b, 2014b, 2015; Moretti et al. 2014; Muraveva et al. 2015). In these papers, we provided relevant results on the calibration of the distance scale for all these important standard candles.

The scope of this paper is to present the results for the CCs in the SMC after four years of VMC observations. The SMC is known to host more than $4500 \mathrm{CCs}$, according to the OGLE III (Soszyński et al. 2010) and EROS 2 (Tisserand et al. 2007; Kim et al. 2014) surveys. The large number of these pulsators, combined with their characteristic narrow intrinsic PL, PLC, and PW relationships in the NIR, make them perfect tracers to unveil the complex structure of the SMC. Indeed, as outlined

\footnotetext{
14 Visible and Infrared Survey Telescope for Astronomy.

15 However, the possibility that they are old stars that underwent collisional or binary mergers cannot be excluded (see, e.g., Marconi et al. 2004, and references therein).
}

above, the use of NIR relations has several advantages with respect to the optical bands. Thus, the data presented in this paper will allow us to study in more detail compared with past studies the 3D geometry of the galaxy. The results of that analysis will be presented in a forthcoming paper.

This work is organized as follows. Sections 2 and 3 present the observations and the technique used to fit the CC light curves, respectively. Section 4 shows the color-magnitude diagrams and peak-to-peak amplitudes; in Section 5 we illustrate the PL, PLC, and PW relationships obtained for the SMC CCs and the associated results; a brief final Section 6 summarizes the paper.

\section{SMC CLASSICAL CEPHEIDS IN THE VMC SURVEY}

As referred to above, the two survey projects that identified CCs in the SMC are OGLE III (Soszyński et al. 2010) and EROS 2 (Tisserand et al. 2007). The areas covered by the two surveys overlap almost completely, although OGLE III extends more toward the east, whereas EROS 2 covers a small corner in the northwest where OGLE III data is not available (see Figure 4 in Moretti et al. 2014, for a comparison).

In more detail, (Soszyński et al. 2010) reported the identification, the $V, I$ light curves, and the main characteristics (periods, mean magnitudes, etc.) of $4630 \mathrm{CCs}$ in the SMC. The EROS 2 collaboration provided us with a list of CC candidates that was analyzed as described in Moretti et al. (2014) to reject contaminating binaries, resulting in $151 \mathrm{CC}$ candidates. Among these objects, only about 20 were located outside the area investigated by OGLE III. A quick comparison of the PW in the $V, I$ bands ${ }^{16}$ revealed that the EROS 2 CC candidates were severely contaminated by other types of variables (typically Type II Cepheids or Anomalous Cepheids) or by other unknown objects. To avoid including spurious objects in our sample, we decided to use only OGLE III data in the area covered by this survey, and to consider only the 20 EROS 2 $\mathrm{CC}$ candidates in the (small) area covered by this survey but not by OGLE III. After removing from this small sample those objects that were found to lie very far from the OGLE III PW, we ended up with 13 bona fide CC candidates in the EROS 2only field.

In this paper, we present results for the CCs included in 11 tiles (each tile is $1.5 \mathrm{deg}^{2}$ on the sky) completely or nearly completely observed, processed, and cataloged by the VMC survey as of 2015 March 9 (including observations obtained until 2014 September), namely, the tiles SMC 3_3, 3_5, 4_2, 4_3, 4_4, 4_5, 5_2, 5_3, 5_4, 6_3, and 6_5. Figure 1 shows the spatial extent of the VMC tiles across the SMC body. The completed tiles do not cover the entire area surveyed by OGLE III. However, given the high concentration of CCs in the central body of the SMC, the number of pulsators included in the completed VMC tiles is about $90 \%$ of the total OGLE III sample. Table 1 lists the coordinates of the quoted tiles, as well as the number of CCs included in each tile.

In total, we were able to study 4159 objects of the 4630 OGLE III sample. To this number we have to add the $13 \mathrm{CCs}$ from the EROS 2 data, leaving us with a final sample of 4172 CCs. The classification of the investigated pulsators in terms of

\footnotetext{
16 The Wesenheit magnitude in this case is defined as $W(V, I)=V-2.54(V-I)$. Note that EROS 2 observations were carried out using custom $B_{\text {EROS } 2}$ and $R_{\text {EROS } 2}$ filters that can be approximately converted to the Johnson $V, I$ bands using the transformation provided by Tisserand et al. (2007)
} 


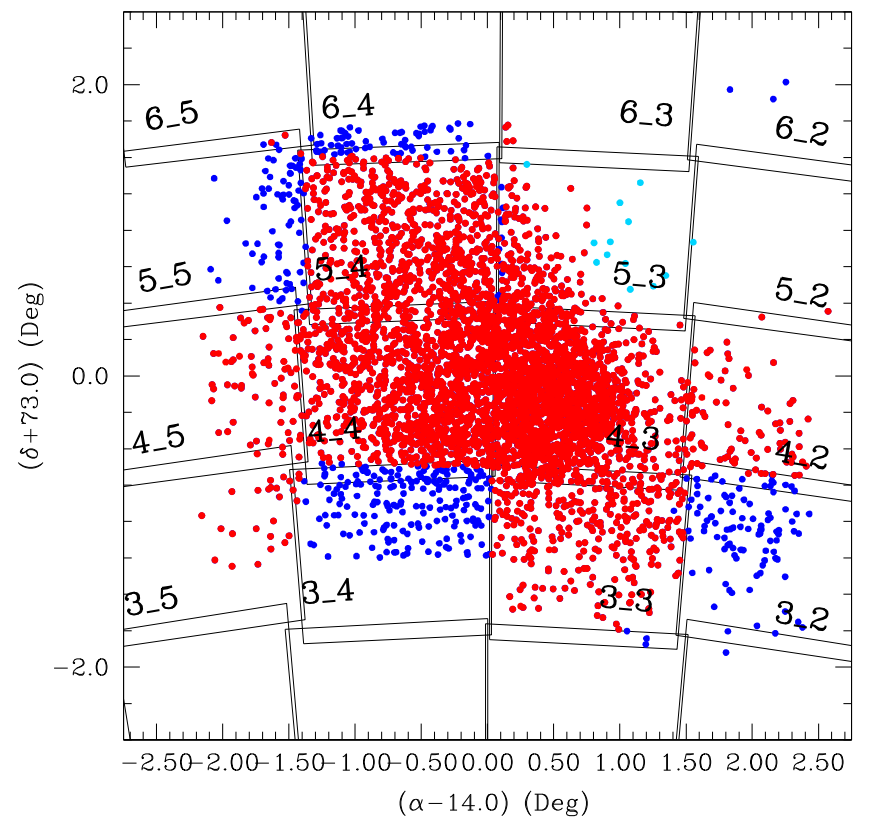

Figure 1. Map of the CCs in the SMC. Red and blue filled circles represent pulsators present in the OGLE III catalog and indicated whether or not they have been observed by the VMC Survey, respectively. Light blue symbols show the 13 Cepheids identified on the basis of the EROS 2 data (Tisserand et al. 2007; Moretti et al. 2014).

Table 1

Number of CCs in Each VMC SMC Tile

\begin{tabular}{|c|c|c|c|}
\hline Tile & $\begin{array}{l}\text { R.A. } \\
\text { hh mm ss.sss }\end{array}$ & $\begin{array}{c}\text { decl. } \\
\circ / 11\end{array}$ & $N$ \\
\hline SMC 3_3 & 004455.896 & $-74 \quad 1242.120$ & 315 \\
\hline SMC 3_5 & 012730.816 & -740049.320 & 25 \\
\hline SMC 4_2 & 002514.088 & -730147.640 & 86 \\
\hline SMC 4_3 & 004514.688 & -730711.280 & 1642 \\
\hline SMC 4_4 & 010519.272 & -730515.360 & 1128 \\
\hline SMC 4_5 & 012511.088 & -725602.760 & 83 \\
\hline SMC 5_2 & 002641.688 & -715635.880 & 2 \\
\hline SMC 5_3 & 004532.232 & -720140.080 & 197 \\
\hline SMC 5_4 & 010426.112 & -715951.000 & 687 \\
\hline SMC 6_3 & 004548.792 & -705609.240 & 4 \\
\hline SMC 6_5 & 012122.560 & -704611.640 & 3 \\
\hline
\end{tabular}

Fundamental (F), First Overtone (FO), Second Overtone (SO), and mixed modes (F/FO, FO/SO, F/FO/SO, and $\mathrm{FO} / \mathrm{SO} /$ TO, where TO stands for Third Overtone) is shown in Table 2.

A general description of the observing strategy of the VMC survey can be found in Cioni et al. (2011). The procedures adopted to study the variable stars were discussed in detail by Ripepi et al. (2012a, 2012b, 2014b, 2015), Moretti et al. (2014). However, it is worth recalling that the VMC $K_{\mathrm{s}}$-band time-series observations were scheduled to span 13 separate epochs distributed over several consecutive months. This observing strategy permits one to achieve well-sampled light curves for different types of variable stars, including RR Lyrae variables and Cepheids of all types. As for the $Y$ and $J$ bands, the nominal number of epochs is four (two of these epochs are obtained with half exposure time) and may be acquired during the same night given that monitoring in these filters was not planned. However, a few additional epochs are usually available for each tile (especially in the $K_{\mathrm{s}}$-band), because some observing blocks were executed outside of our
Table 2

Number of CCs for Each Different Mode of Pulsation

\begin{tabular}{lcccccc}
\hline \hline $\mathrm{F}$ & $\mathrm{FO}$ & $\mathrm{SO}$ & $\mathrm{F} / \mathrm{FO}$ & $\mathrm{FO} / \mathrm{SO}$ & $\mathrm{F} / \mathrm{FO} / \mathrm{SO}$ & $\mathrm{FO} / \mathrm{SO} / \mathrm{TO}$ \\
\hline 2377 & 1472 & 74 & 52 & 196 & 2 & 1 \\
\hline
\end{tabular}

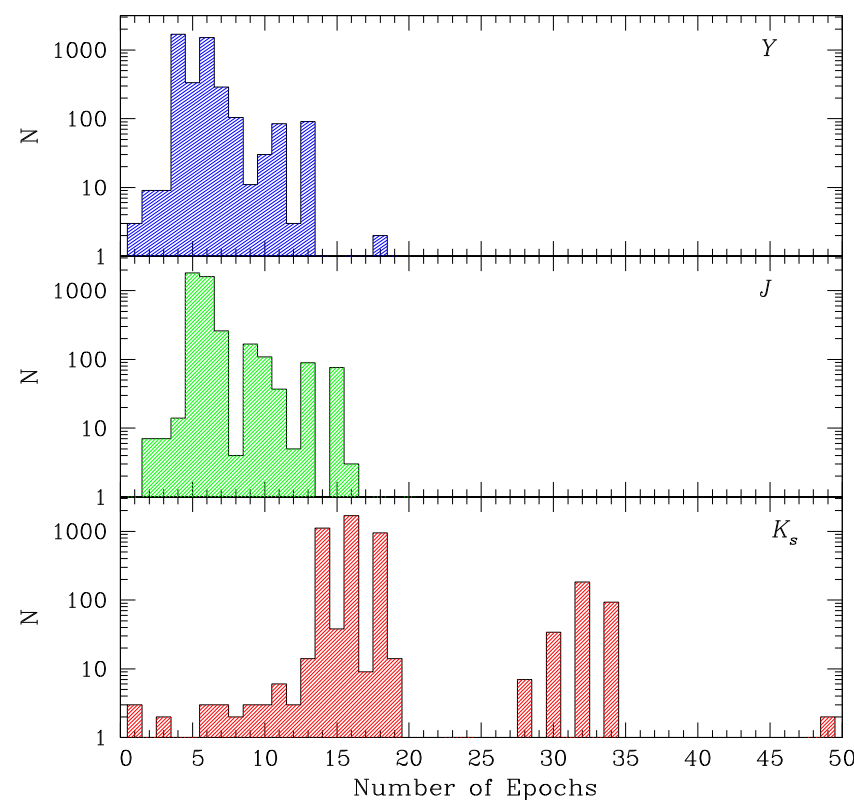

Figure 2. Histogram of the numbers of epochs in each photometric band.

specifications (typically for seeing values exceeding 0.8-1.0 arcsec), but the data were still useful since the CCs are relatively bright. In addition, there is a small overlap between the tiles. Consequently, the CCs present in multiple tiles possess at least twice the scheduled number of epochs. Given the high concentration of CCs in the contiguous tiles SMC 4_3, 4_4, 5_3, and 5_4 (see Figure 1), we have more than 320 CCs whose light curves contain more than 28 phase points. This is also shown in the bottom panel of Figure 2, where the bimodal distribution of epochs in the $K_{\mathrm{s}}$ band is clear. From the figure, note that there are a few dozen stars with fewer than 13 epochs in $K_{\mathrm{s}}$. This can happen when the sources are located in underexposed areas and/or are affected by bright neighbors or bad pixels. We were still able to analyze these stars thanks to our template-fitting procedure (see Section 3).

The same considerations hold for the $Y$ and $J$ bands, whose epoch distributions are shown in the top and middle panels of Figure 2, respectively. In this case, the number of CCs with more than 10 epochs is 213 and 321 in the $Y$ and $J$ bands, respectively. Similarly, the number of CCs with more than five epochs is 2121 and 2343 in $Y$ and $J$, respectively.

The VMC data were processed with the pipeline (Irwin et al. 2004) of the VISTA Data Flow System (VDFS, Emerson et al. 2004) and the photometry is in the VISTA photometric system $($ Vegamag $=0)$. The time-series data analyzed in this work were downloaded from the VISTA Science Archive ${ }^{17}$ (VSA, Cross et al. 2012). Details about the data reduction can be found in the aforementioned papers. However, we briefly recall that (i) the pipeline applies a correction to the photometry of stars close to the saturation limit (Irwin 2009). This task is very

${ }^{17}$ http://horus.roe.ac.uk/vsa/index.html 

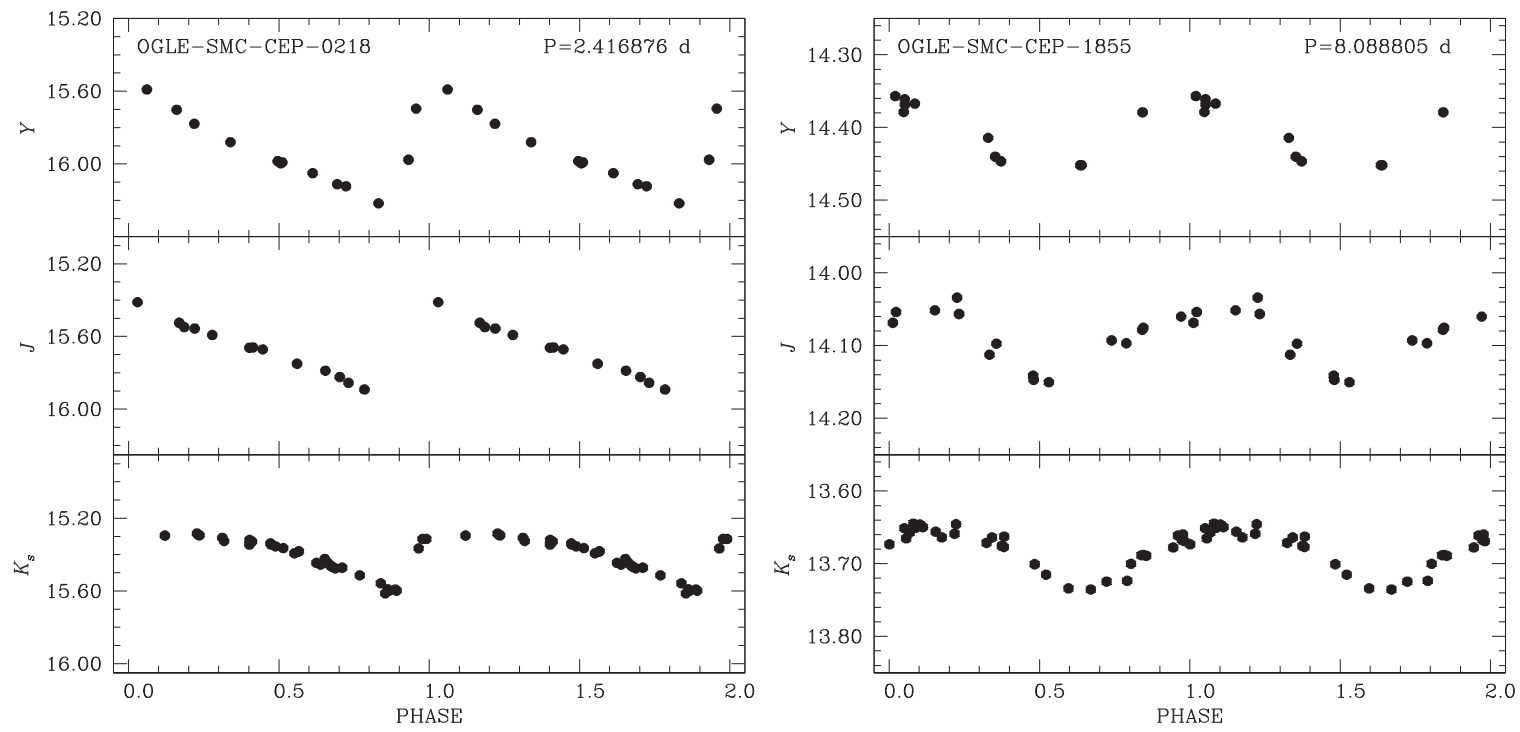

Figure 3. $Y, J, K_{\mathrm{s}}$ light curves for the labelled Cepheids. The errors are of similar size or smaller than the size of the symbols. Note the quality of the data and the change in amplitude and shape of the light curve going from the $Y$ to the $K_{\mathrm{s}}$ bands.

useful, because long-period $\mathrm{CCs}$ are very bright $\left(K_{\mathrm{s}} \sim 12-13\right) \mathrm{mag}$. The time-series photometry of these variables takes advantage of the VDFS capability to deal with the images for saturation, although the corrections applied do not always guarantee a full recovery of the data. (ii) The VSA processing produces quality flags for each star that are valuable to understand if the images have problems. This information is important for the following analysis.

To obtain the $Y, J$, and $K_{\mathrm{S}}$ light curves, the OGLE III (and EROS 2) catalog(s) of CCs described above were crosscorrelated against the VMC catalog, taking all counterparts from the OGLE III and EROS 2 positions, regardless of their separation on the sky. About $95 \%$ of the objects have positions in agreement with those measured by OGLE III and EROS 2 within less than $0 . " 1$. Among the remaining 186 stars, 67 have a separation larger than $0 . " 5$ and are likely misidentifications. We will come back to these objects below.

The typical quality of the light curves obtained is illustrated in Figure 3 for two $\mathrm{F}$ pulsators with very different periods. VMC photometry for the 4172 stars analyzed in this work is reported in Table 3. The complete version of the table is available online at the journal site.

\section{TEMPLATE-FITTING PROCEDURE}

Given the large number of light curves to analyze, it was convenient to find an automatic way to process the data. Our aim is to obtain an analytical or empirical model light curve that fits the observed one. This model can subsequently be used to measure the mean magnitude and the peak-to-peak amplitude for each variable. The usual way to carry out such a task is to use truncated Fourier series, adding as many harmonics as needed to obtain a good fit to the data (Schaltenbrand \& Tammann 1971). However, this kind of approach would not be useful in our case because the presence
Table 3

$Y, J$, and $K_{\mathrm{s}}$ Time Series Photometry for the CCs Investigated in this Paper

\begin{tabular}{|c|c|c|}
\hline HJD-2400000 & $Y$ & $\sigma_{Y}$ \\
\hline 55492.59731 & 19.076 & 0.040 \\
\hline 55492.63328 & 19.130 & 0.040 \\
\hline 55497.70319 & 19.116 & 0.047 \\
\hline 55539.61969 & 19.114 & 0.051 \\
\hline HJD-2400000 & $J$ & $\sigma_{J}$ \\
\hline 55493.58975 & 18.788 & 0.041 \\
\hline 55493.62870 & 18.830 & 0.039 \\
\hline 55495.55177 & 18.860 & 0.063 \\
\hline 55539.64087 & 18.912 & 0.060 \\
\hline 55778.75378 & 18.817 & 0.052 \\
\hline HJD-2400000 & $K_{\mathrm{s}}$ & $\sigma_{K_{\mathrm{s}}}$ \\
\hline 55493.78892 & 18.641 & 0.099 \\
\hline 55495.57575 & 18.699 & 0.168 \\
\hline 55495.68566 & 18.688 & 0.111 \\
\hline 55497.72461 & 18.776 & 0.153 \\
\hline 55538.62081 & 18.681 & 0.125 \\
\hline 55549.58532 & 18.777 & 0.135 \\
\hline 55769.75425 & 18.697 & 0.126 \\
\hline 55778.77517 & 18.683 & 0.169 \\
\hline 55791.76203 & 18.706 & 0.110 \\
\hline 55818.73171 & 18.807 & 0.148 \\
\hline 55820.67458 & 18.484 & 0.088 \\
\hline 55879.55553 & 18.542 & 0.111 \\
\hline 55880.61929 & 18.737 & 0.137 \\
\hline 55900.57090 & 18.674 & 0.115 \\
\hline 56130.79475 & 18.704 & 0.116 \\
\hline 56173.70295 & 18.712 & 0.136 \\
\hline 56195.64097 & 18.668 & 0.111 \\
\hline 56223.54177 & 18.704 & 0.125 \\
\hline
\end{tabular}

Note. The data below refer to the variable OGLE-SMC-CEP-2476.

(This table is available in its entirety in machine-readable form.) 
of significant gaps in the light curve would lead to strong and unrealistic oscillations in the Fourier series.

Hence, we decided to use template light curves to fit the data. Following the pioneering work by Freedman (1988), templates to fit CC light curves based on only a few epochs in the NIR bands have already been presented and used by Soszyński et al. (2005) and Inno et al. (2013, 2015). The typical approach in these studies consists of the following steps: (1) adopting templates constructed based on well-sampled CC $J, H$, and $K_{\mathrm{s}}$ light curves (Galactic or MC objects); (2) scaling the template amplitude using fixed amplitude ratios (e.g., $A(J) / A(V)$, with some dependence on period); (3) adopting literature period and epoch of maximum light to phase-match the template and the observed data. This technique is valuable, since it allows one to obtain an estimate of the average magnitude of a CC based on just one or two observed phase points. At the same time, given the uncertainties on the amplitude ratios and on the ephemerides, these estimates can easily be affected by errors as large as 5\% (see also Section 3.3), despite the quite low amplitudes of the light curves in the NIR bands.

Our approach is fundamentally different from that outlined above (e.g., by Inno et al. 2013). Indeed, the availability of an average of $\sim 5.7,6.3$, and 16.7 phase points in $Y, J$, and $K_{\mathrm{s}}$, respectively, allows us to safely rescale our templates in amplitude and phase match them using our observations directly. This procedure eliminates most of the uncertainties of the "classical" template method, because we do not have to rely on any fixed amplitude ratio to scale the templates in amplitude, nor do we have to use the literature epoch of maximum as reference to phase match the template and the observed data.

\subsection{Template Construction}

The first step of our procedure was the construction of the templates. To this end, we visually inspected a large number of light curves, trying to select those with the most often recurring shapes, and at the same time, those exhibiting precise light curves. Particular care was devoted to covering a broad range of periods. This search was rather simple in $K_{\mathrm{s}}$, since in this band we have dozens of well-sampled and precise light curves for any period. However, we had fewer choices in $Y$ and $J$, given the much smaller number of well-sampled light curves in those filters.

At the end of this process, we concluded that a set of eight different templates for each band could reproduce the variety of shapes exhibited by the observed light curves.

Our templates were constructed as truncated Fourier series of the form

$$
m(\phi)=a_{0}+\sum_{k=1}^{N}\left[a_{k} \cos \left(2 \pi k \phi+\Phi_{k}\right)\right],
$$

where $m$ is the magnitude, $\phi$ are the phases of the template light curves, $a_{0}$ is the zero point, which is zero by definition, $N$ is the number of terms of the series, and $a_{k}$ and $\Phi_{k}$ are the amplitudes and phases of each term of the series, respectively. The first step consisted of fitting the selected observed light curves with splines in order to have smooth, densely sampled curves to be passed to the Fourier-series fitting program. This was needed to avoid spurious oscillations in the Fourier-series fit due to possible small gaps or undersampling at maximum/minimum of the light curves. This way, we actually constructed six of the eight different templates adopted for each filter. They are listed in Table 4, from T3 to T8. As for the two remaining templates, T1 simply consists of a pure cosine function for all filters (which is why the T1 template is not included in the table), while T2 reproduces a smooth curve which can often be observed in all of the $Y, J$, and $K_{\mathrm{s}}$ bands for a broad range of periods (see T2 in Table 4). The shapes of the eight templates in all three filters are shown in Figure 4.

\subsection{Template Fitting}

The template-fitting procedure includes the following steps.

1. Scaling the templates with amplitude ratios, e.g., $A\left(K_{\mathrm{s}}\right) /$ $A(I)$, where we take $A(I)$ from the OGLE III survey and the coefficients of these ratios from Soszyński et al. (2005). Similarly, the template is phase-matched with the observations using the ephemerides from OGLE III. The purpose of this step is only to provide a first-guess average magnitude for the target star and, in turn, to remove the most obvious outliers. In practice, we simply estimate the template values at the phases of the observed point and calculate the average difference between observed and calculated values, which is the approximate mean magnitude of the star.

2. Recalculating the template by varying its initial phase to find the phase shift that minimizes (by means of a leastsquares fit) the difference data-template. This step provides an improved average magnitude (of the order of a few hundredths of mag).

3. Recalculating the template by varying its amplitude to find the amplitude scaling that minimizes (by means of a least-squares fit) the difference data-template. This step provides a further improvement of the average magnitude (again, a few hundredths of mag).

4. Fine-tuning outlier removal (at $2 \sigma$ and $3 \sigma$ levels in $K_{\mathrm{s}}$ and in $Y, J$, respectively; the difference is because in $Y, J$ we have many fewer phase points than in $K_{\mathrm{s}}$ and, hence, we can simply remove obvious outliers) and final averagemagnitude calculation (in intensity).

This procedure is applied to each template (in each band). Next, we need a tool to choose the template that optimally represents the data. After several trials and visual inspections of the resulting fits, we devised two main useful diagnostics. The first is the usual $\chi^{2}$ minimization, defined as

$$
\chi^{2}=\frac{1}{N} \sum_{\phi=0}^{1}\left(\frac{m(\phi)_{\mathrm{obs}}-m(\phi)_{\mathrm{temp}}}{\sigma\left(m(\phi)_{\mathrm{obs}}\right)}\right)^{2},
$$

where $N$ is the total number of phase points, $m_{\mathrm{obs}}$ and $m_{\mathrm{temp}}$ are the magnitudes of the observed and computed light curves, respectively, and $\sigma\left(m_{\text {obs }}\right)$ is the magnitude uncertainty per phase point.

The second criterion was devised empirically to take into account the fact that the smallest residuals can result from application of the wrong template simply because the outlierremoval process is too aggressive. Thus, we designed a Goodness (or $G$ ) parameter, defined as

$$
G=\operatorname{int}\left[\left(\frac{1}{\sigma}\right)^{2}\left(\frac{N_{\mathrm{U}}}{N_{\mathrm{T}}}\right)^{4} 10^{6}\right],
$$


Table 4

Fourier Parameters Adopted to Construct the Templates in the VISTA $Y, J, K_{\mathrm{s}}$ Bands

\begin{tabular}{|c|c|c|c|c|c|c|c|}
\hline Parameter & $\mathrm{T} 2$ & $\mathrm{~T} 3$ & $\mathrm{~T} 4$ & T5 & T6 & $\mathrm{T} 7$ & $\mathrm{~T} 8$ \\
\hline \multicolumn{8}{|c|}{$Y$-Band } \\
\hline$a_{1}$ & 0.49260 & 0.12614 & 0.12888 & 0.20863 & 0.20097 & 0.17442 & 0.15502 \\
\hline$a_{2}$ & 0.14500 & 0.03315 & 0.07549 & 0.11718 & 0.09804 & 0.05488 & 0.03855 \\
\hline$a_{3}$ & 0.04100 & 0.01090 & 0.04152 & 0.05601 & 0.04747 & 0.02273 & 0.02314 \\
\hline$a_{4}$ & 0.01000 & 0.00448 & 0.01609 & 0.02200 & 0.01831 & 0.01211 & 0.00984 \\
\hline$a_{5}$ & 0.00000 & 0.00209 & 0.00580 & 0.00765 & 0.00370 & 0.00640 & 0.00483 \\
\hline$a_{6}$ & 0.00000 & 0.00124 & 0.00000 & 0.00253 & 0.00190 & 0.00345 & 0.00146 \\
\hline$a_{7}$ & 0.00000 & 0.00065 & 0.00000 & 0.00026 & 0.00276 & 0.00171 & 0.00078 \\
\hline$a_{8}$ & 0.00000 & 0.00035 & 0.00000 & 0.00044 & 0.00173 & 0.00081 & 0.00054 \\
\hline$a_{9}$ & 0.00000 & 0.00022 & 0.00000 & 0.00008 & 0.00054 & 0.00031 & 0.00041 \\
\hline$a_{10}$ & 0.00000 & 0.00017 & 0.00000 & 0.00027 & 0.00024 & 0.00010 & 0.00022 \\
\hline$\phi_{1}$ & 1.40800 & 5.75860 & 2.52701 & 1.50221 & 0.72710 & 5.27327 & 5.93367 \\
\hline$\phi_{2}$ & 2.52800 & 3.65703 & 3.36472 & 1.50458 & 6.08919 & 3.02993 & 4.57391 \\
\hline$\phi_{3}$ & 3.55200 & 1.23267 & 4.18291 & 1.34113 & 5.11517 & 1.14020 & 3.82891 \\
\hline$\phi_{4}$ & 4.43800 & 5.38378 & 5.03844 & 0.97634 & 4.16420 & 5.46810 & 1.84753 \\
\hline$\phi_{5}$ & 0.00000 & 3.36130 & 5.72830 & 0.30153 & 3.35092 & 3.52010 & 1.18378 \\
\hline$\phi_{6}$ & 0.00000 & 1.20985 & 0.00000 & 5.90041 & 5.31224 & 1.54636 & 4.81460 \\
\hline$\phi_{7}$ & 0.00000 & 5.24591 & 0.00000 & 0.00420 & 4.58734 & 5.86030 & 5.81304 \\
\hline$\phi_{8}$ & 0.00000 & 3.03051 & 0.00000 & 1.40454 & 3.82023 & 3.83808 & 0.48183 \\
\hline$\phi_{9}$ & 0.00000 & 1.01212 & 0.00000 & 0.34470 & 3.26788 & 1.67678 & 3.77426 \\
\hline$\phi_{10}$ & 0.00000 & 5.12881 & 0.00000 & 3.91317 & 4.72011 & 5.36878 & 4.02321 \\
\hline \multicolumn{8}{|c|}{$J$-Band } \\
\hline$a_{1}$ & 0.49260 & 0.10773 & 0.12934 & 0.10873 & 0.12532 & 0.12790 & 0.15502 \\
\hline$a_{2}$ & 0.14500 & 0.01461 & 0.06480 & 0.04565 & 0.03672 & 0.05709 & 0.03855 \\
\hline$a_{3}$ & 0.04100 & 0.02933 & 0.03052 & 0.02487 & 0.00939 & 0.00983 & 0.02314 \\
\hline$a_{4}$ & 0.01000 & 0.00196 & 0.01328 & 0.00825 & 0.00303 & 0.00215 & 0.00984 \\
\hline$a_{5}$ & 0.00000 & 0.00177 & 0.00547 & 0.00220 & 0.00170 & 0.00151 & 0.00483 \\
\hline$a_{6}$ & 0.00000 & 0.00010 & 0.00254 & 0.00091 & 0.00020 & 0.00072 & 0.00146 \\
\hline$a_{7}$ & 0.00000 & 0.00013 & 0.00135 & 0.00018 & 0.00012 & 0.00021 & 0.00078 \\
\hline$a_{8}$ & 0.00000 & 0.00011 & 0.00061 & 0.00057 & 0.00040 & 0.00002 & 0.00054 \\
\hline$a_{9}$ & 0.00000 & 0.00004 & 0.00023 & 0.00046 & 0.00023 & 0.00003 & 0.00041 \\
\hline$a_{10}$ & 0.00000 & 0.00004 & 0.00019 & 0.00031 & 0.00024 & 0.00006 & 0.00022 \\
\hline$\phi_{1}$ & 1.40800 & 0.22905 & 2.72672 & 5.39342 & 1.09258 & 5.12094 & 5.93367 \\
\hline$\phi_{2}$ & 2.52800 & 4.86405 & 4.06800 & 3.37087 & 0.70885 & 2.95240 & 4.57391 \\
\hline$\phi_{3}$ & 3.55200 & 3.12523 & 5.42088 & 1.47564 & 0.70180 & 0.78702 & 3.82891 \\
\hline$\phi_{4}$ & 4.43800 & 1.79845 & 0.59352 & 5.60246 & 1.22024 & 5.53095 & 1.84753 \\
\hline$\phi_{5}$ & 0.00000 & 1.00316 & 2.17713 & 2.89183 & 1.40527 & 2.77300 & 1.18378 \\
\hline$\phi_{6}$ & 0.00000 & 5.97019 & 3.76033 & 0.28963 & 0.69902 & 0.39488 & 4.81460 \\
\hline$\phi_{7}$ & 0.00000 & 5.69884 & 5.19037 & 0.47083 & 5.83421 & 5.20192 & 5.81304 \\
\hline$\phi_{8}$ & 0.00000 & 5.29476 & 0.22745 & 5.03774 & 5.32133 & 3.10882 & 0.48183 \\
\hline$\phi_{9}$ & 0.00000 & 2.24848 & 1.14384 & 2.56761 & 5.91469 & 2.69578 & 3.77426 \\
\hline$\phi_{10}$ & 0.00000 & 2.92779 & 2.44216 & 6.20944 & 5.71899 & 5.71612 & 4.02321 \\
\hline \multicolumn{8}{|c|}{$K_{\mathrm{s}}-$ Band } \\
\hline$a_{1}$ & 0.49260 & 0.11057 & 0.18142 & 0.10520 & 0.15750 & 0.10319 & 0.10789 \\
\hline$a_{2}$ & 0.14500 & 0.04102 & 0.02969 & 0.03653 & 0.02199 & 0.03305 & 0.04682 \\
\hline$a_{3}$ & 0.04100 & 0.01533 & 0.00711 & 0.01983 & 0.00921 & 0.01029 & 0.02355 \\
\hline$a_{4}$ & 0.01000 & 0.00440 & 0.00214 & 0.01085 & 0.00367 & 0.00155 & 0.01431 \\
\hline$a_{5}$ & 0.00000 & 0.00089 & 0.00302 & 0.00550 & 0.00224 & 0.00001 & 0.00800 \\
\hline$a_{6}$ & 0.00000 & 0.00078 & 0.00146 & 0.00259 & 0.00119 & 0.00011 & 0.00448 \\
\hline$a_{7}$ & 0.00000 & 0.00048 & 0.00051 & 0.00127 & 0.00064 & 0.00032 & 0.00232 \\
\hline$a_{8}$ & 0.00000 & 0.00004 & 0.00069 & 0.00079 & 0.00036 & 0.00033 & 0.00088 \\
\hline$a_{9}$ & 0.00000 & 0.00016 & 0.00016 & 0.00058 & 0.00028 & 0.00018 & 0.00028 \\
\hline$a_{10}$ & 0.00000 & 0.00014 & 0.00049 & 0.00045 & 0.00021 & 0.00000 & 0.00000 \\
\hline$\phi_{1}$ & 1.40800 & 1.27522 & 1.10728 & 1.31803 & 1.57541 & 4.75055 & 1.48634 \\
\hline$\phi_{2}$ & 2.52800 & 1.80953 & 2.41492 & 2.06525 & 2.75238 & 2.11317 & 2.14552 \\
\hline$\phi_{3}$ & 3.55200 & 2.46781 & 2.77198 & 2.84018 & 4.16989 & 5.38840 & 2.71326 \\
\hline$\phi_{4}$ & 4.43800 & 2.94883 & 2.45223 & 3.61468 & 5.36458 & 2.83264 & 3.39001 \\
\hline$\phi_{5}$ & 0.00000 & 2.46814 & 2.58196 & 4.34756 & 0.48850 & 4.55134 & 3.97662 \\
\hline$\phi_{6}$ & 0.00000 & 1.69940 & 3.83981 & 4.99929 & 2.10232 & 1.60734 & 4.48671 \\
\hline$\phi_{7}$ & 0.00000 & 2.00844 & 2.89989 & 5.50476 & 3.90737 & 5.88377 & 5.06340 \\
\hline$\phi_{8}$ & 0.00000 & 2.26548 & 4.29762 & 5.96834 & 5.73100 & 3.05768 & 5.44150 \\
\hline
\end{tabular}


Table 4

(Continued)

\begin{tabular}{|c|c|c|c|c|c|c|c|}
\hline Parameter & $\mathrm{T} 2$ & $\mathrm{~T} 3$ & $\mathrm{~T} 4$ & $\mathrm{~T} 5$ & T6 & $\mathrm{T} 7$ & $\mathrm{~T} 8$ \\
\hline$\phi_{9}$ & 0.00000 & 6.16960 & 3.43667 & 0.23028 & 1.22432 & 0.08505 & 5.61231 \\
\hline$\phi_{10}$ & 0.00000 & 0.16239 & 4.88214 & 0.80864 & 2.94561 & 4.77334 & 0.00000 \\
\hline
\end{tabular}

Note. Note that the template starts from 2 because Template 1 (T1) is a simple cosine function.

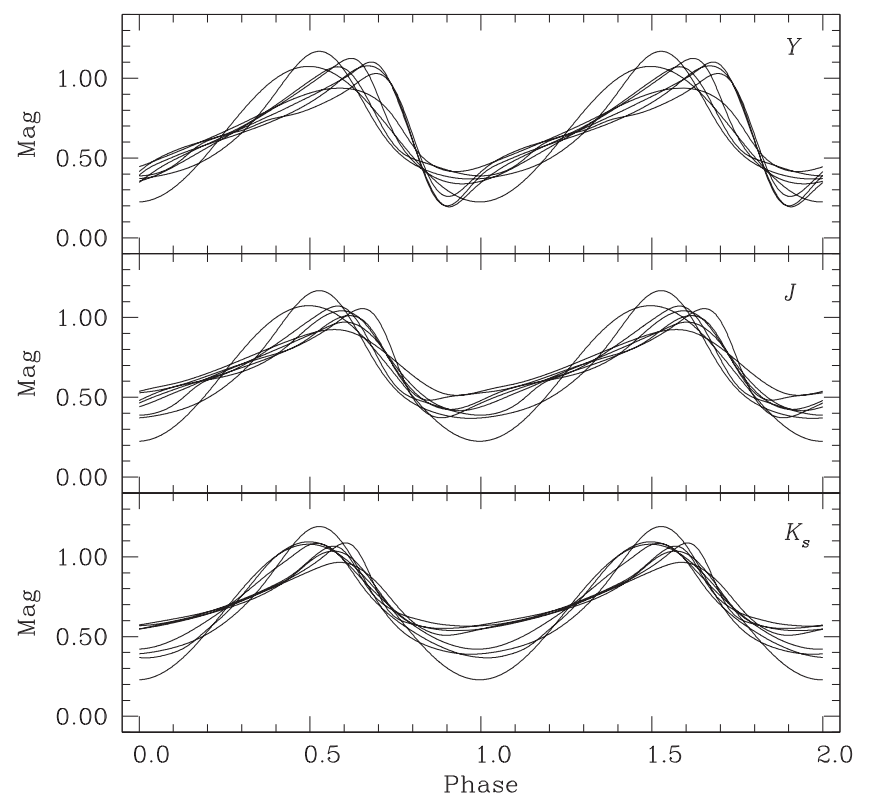

Figure 4. Adopted templates in the $Y J K_{\mathrm{s}}$ bands.

where $\sigma$ is the rms of the fit and $N_{\mathrm{U}}$ and $N_{\mathrm{T}}$ are the numbers of phase points used in the fit and the total number of phase points, respectively. By definition, the first factor of $G$ tends to favor templates that give the smallest rms values, while the second factor favors those that remove the lowest number of outliers. The balance of these two features yields, in general, an automatic decision about the best templates that is in agreement with visual inspection of the fitting procedure. The value of $G$ can be used not only to choose the best template, but also as a more general indicator of the quality of the data and of the relative fitting procedure. Indeed, in general high values of $G$ (in our case, typically $G>100$ ) mean good data (and good fits), while lower values usually indicate significant scatter in the light curves. Extremely high values of $G$ (i.e., $G>10,000$ ) are also rather suspect because non-variable stars, exhibiting completely flat light curves (which happens, for example, when non-variable stars are considered owing to a coordinate missmatch with OGLE III Cepheid data) are expected to yield extremely high values of $G$. Not surprisingly, the $G$ parameter is anti-correlated with the corresponding $\chi^{2}$ value: the higher $G$ is, the lower the $\chi^{2}$ becomes. An example of our templatefitting procedure can be found in Figure 5 (note that in this case the best template is T4), while the anti-correlation between $G$ and $\chi^{2}$ is shown in Figure 6.

\subsection{Monte Carlo Simulations}

As an additional check of the reliability of the templatefitting procedure, and to estimate in a more quantitative way the precision achieved, we decided to use extensive Monte Carlo simulations. In practice, for each star (and for each filter), 100 different mock time series were created on the basis of the observed data, to which Gaussian noise was added with $\sigma$ s corresponding to the average uncertainty on the phase points for the star of interest (different $\sigma$ s were calculated for different filters). The template-fitting procedure outlined above was hence repeated 100 times and the average magnitude and rms were calculated. We then compared these quantities with those calculated from the observed data. The results of this exercise are summarized in Figures 7-9. The top left-hand panels in each figure show the difference between the magnitude obtained with the best-fitting template $\left(Y^{\text {best }}, J^{\text {best }}, K_{\mathrm{s}}^{\text {best }}\right)$ applied to the actual data and that resulting from averaging over the 100 mock light curves $\left(\left\langle Y^{\text {mock }}\right\rangle,\left\langle J^{\text {mock }}\right\rangle,\left\langle K_{\mathrm{s}}^{\text {mock }}\right\rangle\right)$. Similarly, the bottom left-hand panels show the rms of $Y^{\text {mock }}, J^{\text {mock }}$, $K_{\mathrm{s}}^{\text {mock }}$ as a function of $Y^{\text {best }}, J^{\text {best }}, K_{\mathrm{s}}^{\text {best }}$. These figures testify to the high precision reached in the $K_{\mathrm{s}}$ band, where $84 \%$ and $99 \%$ of the stars have errors on the intensity-averaged magnitudes of $\leqslant 0.01$ and $\leqslant 0.02 \mathrm{mag}$ respectively. Only $1 \%$ and $0.1 \%$ of the CCs analyzed here have uncertainties $>0.02$ and $>0.05 \mathrm{mag}$, respectively. The results are less favorable in the $J$ band and even worse in $Y$. In fact, in these bands the corresponding percentages drop to $(68 \%, 93 \%, 7 \%$, and $0.8 \%)$ and $(56 \%$, $78 \%, 22 \%$, and $5.5 \%$ ) in the $J$ and $Y$ bands, respectively. The worse results in $Y$ are mainly due to the fact that (a) the peak-topeak amplitude in this filter is significantly larger than that in the $J$ band (consequently, it is more difficult to estimate the average magnitudes from undersampled light curves) and (b) we have, on average, fewer phase points in $Y$ than in $J(\sim 5.6$ versus $\sim 6.3$ ).

The top and bottom right-hand panels in Figures 7-9 display essentially the same results as the panels on the left, but for the peak-to-peak amplitudes instead of the intensity-averaged magnitudes. Again, the results for the amplitudes in the $K_{\mathrm{s}}$ band are very good, while the uncertainties become significantly larger for the $J$ and, especially, the $Y$ filters.

On the basis of the Monte Carlo experiments, we decided to assign as uncertainties to the intensity-averaged magnitudes and peak-to-peak amplitudes the values shown in the bottom panels of Figures 7-9.

The light curves and the best-fitting templates found with the procedure outlined in this section are reported in Figures 10-12 for the $Y, J$, and $K_{\mathrm{s}}$ bands, respectively. These figures display the data for a subsample of $27 \mathrm{CCs}$; figures including the full data set of 4172 objects are available in the electronic version of this paper on the journal's website.

Similarly, Table 5 reports the main physical quantities derived on the basis of the fitting procedure, namely, the intensity-averaged magnitude for each variable (and each filter), the peak-to-peak amplitudes, and the relative errors calculated by means of the Monte Carlo experiments.

Finally, we recall that the $Y, J$, and $K_{\mathrm{s}}$ photometry described in this work is defined in the VISTA system. It is possible to 


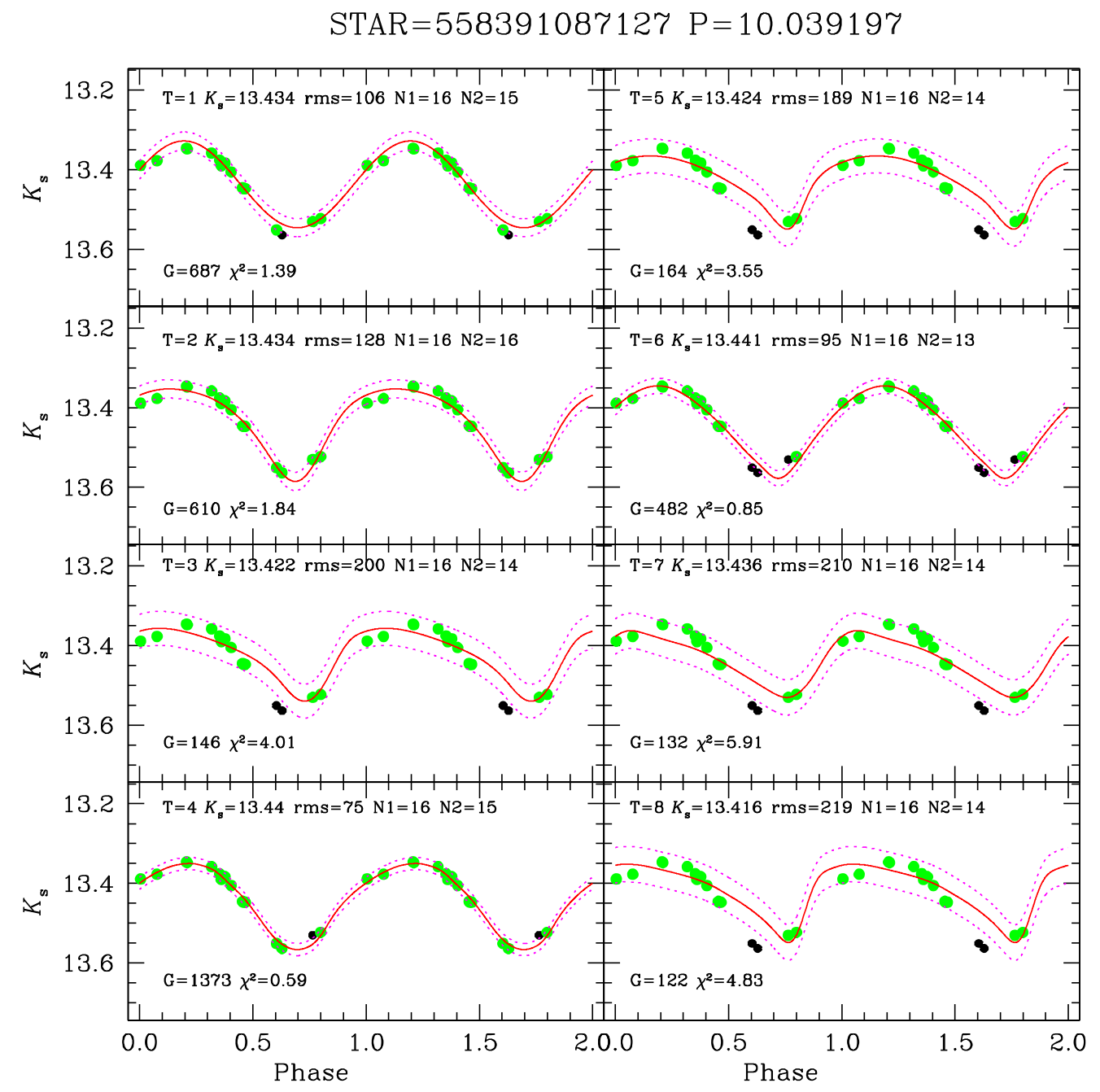

Figure 5. Example of our template-fitting procedure in the $K_{\mathrm{s}}$ band. Each of the eight panels shows the $K_{\mathrm{s}}$ light curve (green and black filled circles show the data points used and rejected in the fitting procedure, respectively). The solid lines are the template curves (labelled with "T" in each panel, with increasing numbers from 1 to 8), properly scaled in amplitude and shifted in phase. The dashed lines represent the $\pm 2 \sigma$ template curves: all data points beyond these lines are marked in black and not included in the fitting procedure. The other labels in each panel are: $K_{\mathrm{s}}=$ mean magnitude of the curve needed to fit the data with the template; rms $=$ root mean square of the fit residuals in mmag; $N 1=$ total number of data points in the light curve; $N 2=$ number of data points used in the fitting procedure; $G=$ goodness parameter (see the text); $\chi^{2}=\chi^{2}$ of the fit (see the text). In this case, the best template is T4.

compare our data with measurements in the widely used 2MASS system (Two Micron All Sky Survey; Skrutskie et al. 2006) after applying the system transformations made available by the Cambridge Astronomy Survey Unit (CASU) ${ }^{18}$ : $\left(J-K_{\mathrm{s}}\right)^{2 \mathrm{M}}=1.081\left(J-K_{\mathrm{s}}\right)^{\mathrm{V}}, J^{2 \mathrm{M}}=J^{\mathrm{V}}+0.07\left(J-K_{\mathrm{s}}\right)^{\mathrm{V}}$, and $K_{\mathrm{s}}^{2 \mathrm{M}}=K_{\mathrm{s}}^{\mathrm{V}}-0.011\left(J-K_{\mathrm{s}}\right)^{\mathrm{V}}$. No transformation is provided in $Y$ since 2MASS did not observe in this filter. However, it is possible to "standardize" the $Y$ band by applying a color equation that, at present, is available only as a function of the $(J-H)$ color (and is therefore of no use to us). A new transformation using the $\left(J-K_{\mathrm{s}}\right)$ bands is being derived by CASU and will be available within a few months.

Since the intrinsic $\langle J\rangle-\left\langle K_{\mathrm{s}}\right\rangle$ colors of the CCs investigated here typically range from $0.1 \mathrm{mag}$ to $0.6 \mathrm{mag}$, the VISTA and 2MASS $K_{\mathrm{s}}$ can be considered equivalent for CCs (see Figure 13) to a very good approximation (better than $\sim 5$ mmag), although the corrections needed in the $J$ band can be significant.

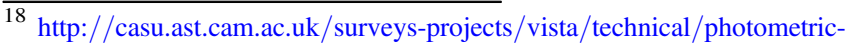
properties
}

\section{AVERAGE MAGNITUDES, COLORS, AND PEAK-TO-PEAK AMPLITUDES}

We constructed color-magnitude diagrams for the entire sample of CC analyzed here, distinguishing them by their different types of pulsation. The results are shown in Figure 13. The middle and right-hand panels of this figure display the comparison in the $K_{\mathrm{s}, 0},\left(J-K_{\mathrm{s}}\right)_{0}$ and $K_{\mathrm{s}, 0},\left(V-K_{\mathrm{s}}\right)_{0}$ planes of the observed data with the theoretical instability strips for $\mathrm{F}$, FO, and SO CCs. The models, calculated for $Z=0.004$ and $Y=0.25$, have been taken from Bono et al. (2000, 2001a, 2001b). We note that the models are in the JHK Johnson system. Thus, we have converted them into the VISTA system, adopting the VISTA-2MASS relations referred to in the previous section, as well as the color transformations available from Bessell \& Brett (1988) and Carpenter (2001). As a result, we obtained the following approximate equations:

$$
\begin{gathered}
K_{\mathrm{s}}^{\mathrm{V}}=K^{\mathrm{J}}+0.007(V-K)^{\mathrm{J}}+0.03(J-K)^{\mathrm{J}}-0.038 \\
\left(V-K_{\mathrm{s}}\right)^{\mathrm{V}}=0.993(V-K)^{\mathrm{J}}-0.03(J-K)^{\mathrm{J}}+0.038 \\
\left(J-K_{\mathrm{s}}\right)^{\mathrm{V}}=0.87(J-K)^{\mathrm{J}}-0.01,
\end{gathered}
$$




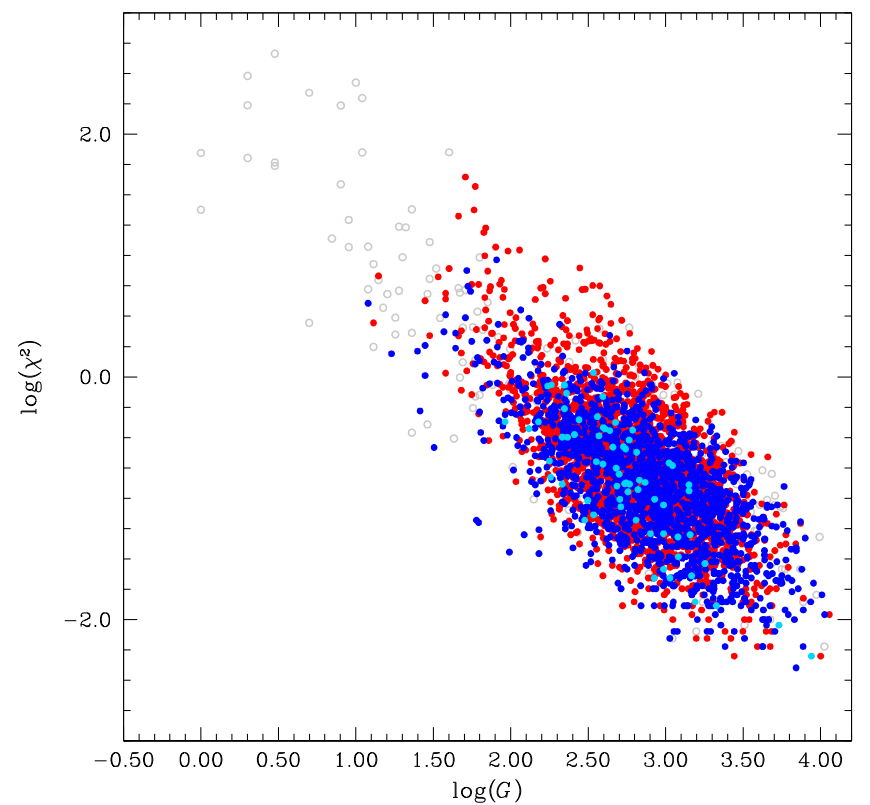

Figure 6. Goodness vs. $\chi^{2}$ in the $K_{\mathrm{s}}$ band for the target CCs. F, FO, and SO pulsators are shown as red, blue, and light blue filled circles, respectively. The gray open circles show the objects excluded from the analysis on the basis of their location near the $\operatorname{PW}\left(K_{\mathrm{s}}, V\right)$ relation (see Section 5).

where the superscripts " $\mathrm{V}$ " and " $\mathrm{J}$ " refer to quantities in the VISTA and Johnson systems, respectively. There is generally good agreement between predicted colors and observations, especially for FO pulsators, while for F pulsators the observed instability strip appears to be larger at low luminosities (i.e., short periods) compared with the predictions.

Additional information can be obtained from the color-color diagrams shown in Figure 14, where the left- and right-hand panels show the $\left(J-K_{\mathrm{s}}, Y-K_{\mathrm{s}}\right)$ and $\left(V-K_{\mathrm{s}}, J-K_{\mathrm{s}}\right)$ planes, respectively. In both cases, arrows represent the reddening vectors, which are almost parallel to the data distribution, making it almost impossible to use these planes to estimate individual reddening values. It is interesting to note the distribution of the rejected stars (empty gray circles), which is markedly elongated toward red colors (especially in the $\left(J-K_{\mathrm{s}}, Y-K_{\mathrm{s}}\right)$ plane). This suggests significant contamination in the $K_{\mathrm{s}}$ band by very red objects, likely red clump or red/ asymptotic giant-branch stars, or bright (early-type) background galaxies. The right-hand panel of Figure 14 shows the theoretical instability strip, now visible as an almost straight line passing, as expected, through the middle of the data distribution. Indeed, at fixed effective temperature, the position in the color-color plane is unequivocally determined by the adopted effective temperature-color transformation. Note that the modest broadening of the data $(\sim 0.07-0.1 \mathrm{mag})$ is due to different contributions, namely, the photometric errors, blending effects, and/or small metallicity differences, but not to reddening effects (see the direction of the reddening vectors in both planes of Figure 14).

Figure 15 shows the period versus peak-to-peak amplitude in the $K_{\mathrm{s}}$ band for the target CCs. As far as we know, this is the first time that such a plane has been exploited with such a statistically significant number of objects in an infrared band. An inspection of the figure reveals the clean separation in amplitude of the three different modes. It is interesting to note that the peculiar shape of the distribution of $\mathrm{F}$ pulsators, with an increase at about $P=10$ days and a maximum around $P=22-24$ days, resembles a similar trend observed in the visual $V$ band for the Galactic CCs (Bono et al. 2000) and is consistent with theoretical predictions (see their Figure 7).

We also looked at the peak-to-peak amplitude ratios for different pulsation modes between the $K_{\mathrm{s}}$ and $I$ bands. These values may be useful for authors who want to use the canonical template-fitting procedure. Figure 16 shows the $R\left(K_{\mathrm{s}}, I\right)=$ $A\left(K_{\mathrm{s}}\right) / A(I)$ ratio versus period for the CCs investigated here. We calculated the ratio between these bands, because our amplitudes are more accurate in $K_{\mathrm{s}}$ relative to $Y$ and $J$ (see Section 3) and OGLE III provides the peak-to-peak amplitudes for all Cepheids investigated here only in the $I$ filter.

Given the rather large scatter in the data (possibly in part due to the presence of binary companions), we decided to average the CCs in period bins, obtaining the light blue and yellow filled circles for $\mathrm{F}$ and FO pulsators, respectively (we did not consider the SO CCs because of their very small amplitudes). An analysis of the averaged data reveals the different behavior of $\mathrm{F}$ and $\mathrm{FO}$ pulsators. $R\left(K_{\mathrm{s}}, I\right)$ is almost constant for $\mathrm{FO}$ pulsators over the full period range, while for $F$ pulsators it is flat only until $P \sim 2.95$ days. For longer periods there is a steep linear increase of $R\left(K_{\mathrm{s}}, I\right)$ with period. Quantitatively, we derived the following equations for $\mathrm{F}$ and FO pulsators:

$$
\begin{aligned}
& R\left(K_{S}, I\right)_{F}=(0.04 \pm 0.01) \log P \\
&+(0.40 \pm 0.01)(P<2.95 \text { days }) \\
& R\left(K_{S}, I\right)_{F}=(0.26 \pm 0.02) \log P \\
&+(0.31 \pm 0.02)(P \geqslant 2.95 \text { days }) \\
& R\left(K_{S}, I\right)_{F O}=(0.39 \pm 0.01) .
\end{aligned}
$$

It is interesting to note that the steep change in slope for $\mathrm{F}$ pulsators occurs at about the same period where we find a break in the PL, PW, and PLC relations (see next section).

A comparison of our results with those in the literature reveals some differences. Indeed, concerning F pulsators only, Soszyński et al. (2005) suggest using constant values of $R\left(K_{S}, I\right)=0.49$ or 0.62 for periods $</ \geqslant \sim 20$ days, respectively. Using Equation (8), for $P \sim 20$ days we obtain $R\left(K_{S}, I\right)=0.65$, which is fully compatible with Soszyński et al. (2005)'s values. However, it is easy to verify that the agreement is worse for different periods. For example, at $P=40$ days, we obtain $R\left(K_{S}, I\right)=0.73$, while at $P=2$ days $R\left(K_{S}, I\right)=0.41$. Taking into account that the Soszyński et al. (2005) results have been derived using Galactic and LMC CCs, it is reasonable to hypothesize that part of the discrepancy between our and their findings can be owing to the different metallicities of the adopted CC samples.

We cannot perform a direct comparison with Inno et al. (2015)'s results, because they only provide the ratio of NIR bands with respect to the $V$ band. However, we can compare the trends versus the periods, since they have different data sets for Galactic+LMC and SMC CCs. As a result, Inno et al. (2015) found a break in $R\left(K_{S}, I\right)$ at a period similar to that of Soszyński et al. (2005). This is in contrast with our results (perhaps this is due to the smaller size of their sample). On the other hand, they found systematically lower $R\left(K_{S}, I\right)$ values for SMC CCs, with respect to the Galactic+LMC variables, in full agreement with our results. 

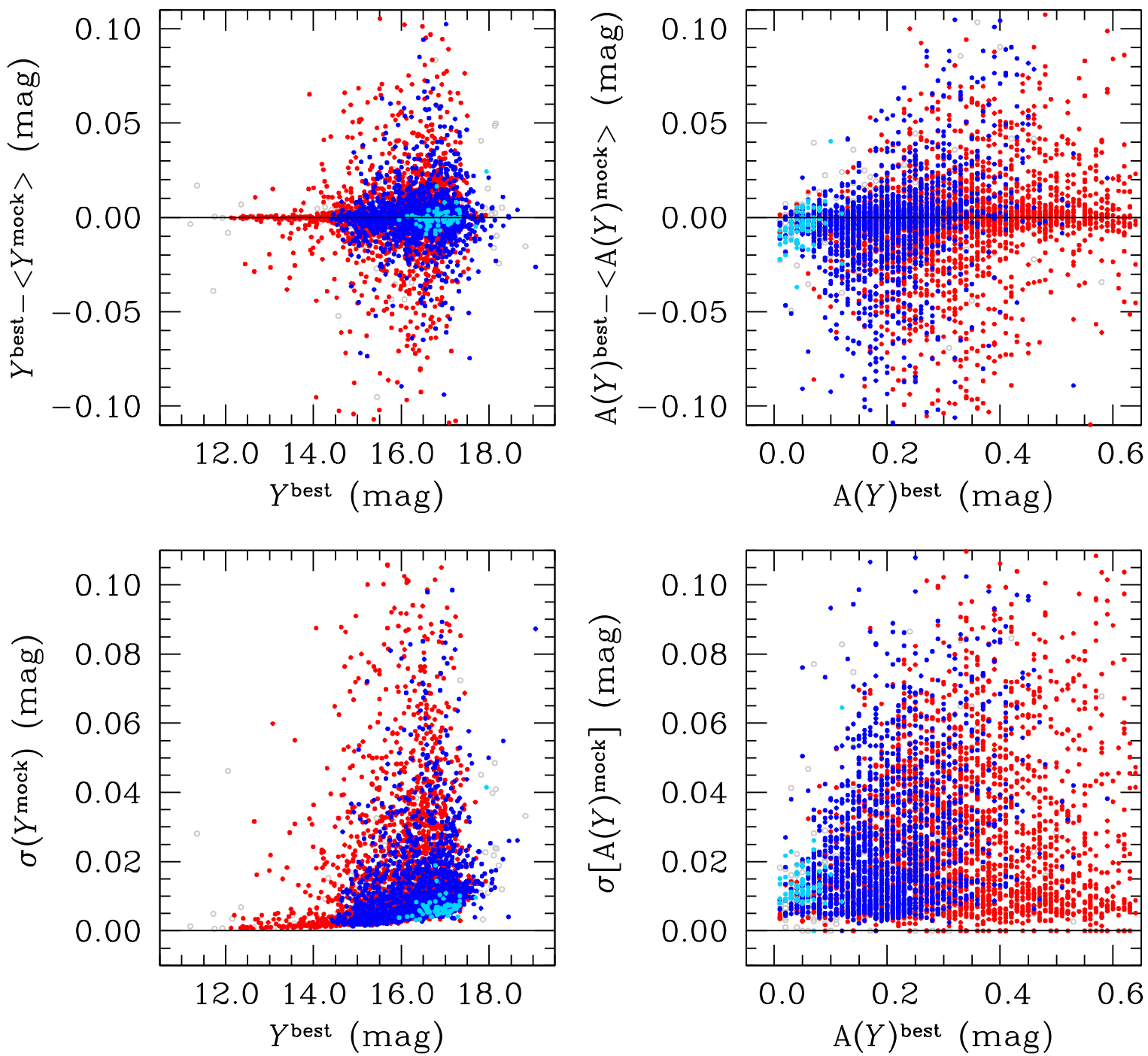

Figure 7. Results from the Monte Carlo experiments in the $Y$ band. The top left-hand panel shows, for each star, the difference between the magnitude obtained with the best-fitting template $\left(Y^{\text {best }}\right)$ applied to the real data and that resulting from averaging over the 100 Monte Carlo experiments $\left(\left\langle Y^{\text {mock }}\right\rangle\right)$. The bottom left-hand panel shows the rms of $Y^{\text {mock }}$ as a function of $Y^{\text {best }}$. The top and bottom right-hand panels are similar to those on the left but display the peak-to-peak amplitudes instead of the magnitudes (in the $Y$ band). The color coding is the same as in Figure 6.

\section{PL, PLC, AND PW RELATIONS}

The data reported in Table 5 allow us to derive several useful relationships, adopting various combinations of magnitudes and colors. In particular, we derived PL relations in $Y, J$, and $K_{\mathrm{s}}$, as well as PW and PLC relations for the following combinations: $\left(K_{\mathrm{s}}, Y-K_{\mathrm{s}}\right),\left(K_{\mathrm{s}}, J-K_{\mathrm{s}}\right)$, and $\left(K_{\mathrm{s}}, V-K_{\mathrm{s}}\right)$.

Before deriving the latter relationships, we have to take account of the reddening. We adopted the extinction maps of Haschke et al. (2011), as we already successfully did in our previous papers (see, e.g., the discussion in Section 3 of Ripepi et al. 2015). The reddening values were converted using the following equations: $E\left(Y-K_{\mathrm{s}}\right)=1.80 E(V-I)$; $E\left(V-K_{\mathrm{s}}\right)=2.24 E(V-I) ; E\left(J-K_{\mathrm{s}}\right)=0.43 E(V-I)($ Cardelli et al. 1989; Kerber et al. 2009; Gao et al. 2013). ${ }^{19}$ The coefficients of the PW relations were calculated in a similar fashion.

\footnotetext{
19 The coefficients used in this paper are consistent with the 2MASS system, to which the VISTA system is related.
}

To derive the relevant relationships for $\mathrm{F}, \mathrm{FO}$, and $\mathrm{SO}$ variables, we adopted equations of the form $M 1_{0}=\alpha+\beta \log P, \quad W(M 1, M 2)=\alpha+\beta \log P, \quad$ and $M 1_{0}=\alpha+\beta \log P+\gamma(M 2-M 1)_{0}$ for the PL, PW, and PLC relations, respectively. Here, $M 1$ and $M 2$ represent two different magnitudes from among $V, Y, J$, and $K_{\mathrm{s}}$. The details about the combinations of magnitudes and colors adopted in this papers can be found in Table 6. In order to use the full sample of pulsators, including the double- or multi-mode CCs, we decided to use them with the period of the dominant mode (e.g., F-mode if the star is an F/FO double-mode pulsator, and so on). This procedure is safe, since from our previous investigation of LMC pulsators (Ripepi et al. 2012b) we know that these objects do not exhibit systematic luminosity differences with respect to single-mode objects.

The next step involved checking for the presence of changes in the slope of the different relationships, as found in previous studies in the literature (see, e.g., Subramanian \& Subramaniam 2015, and references therein). To this aim, we used the PW in $V, K_{\mathrm{s}}$ which was known from our previous investigation of the LMC CCs, to show a small intrinsic dispersion (see, e.g., 

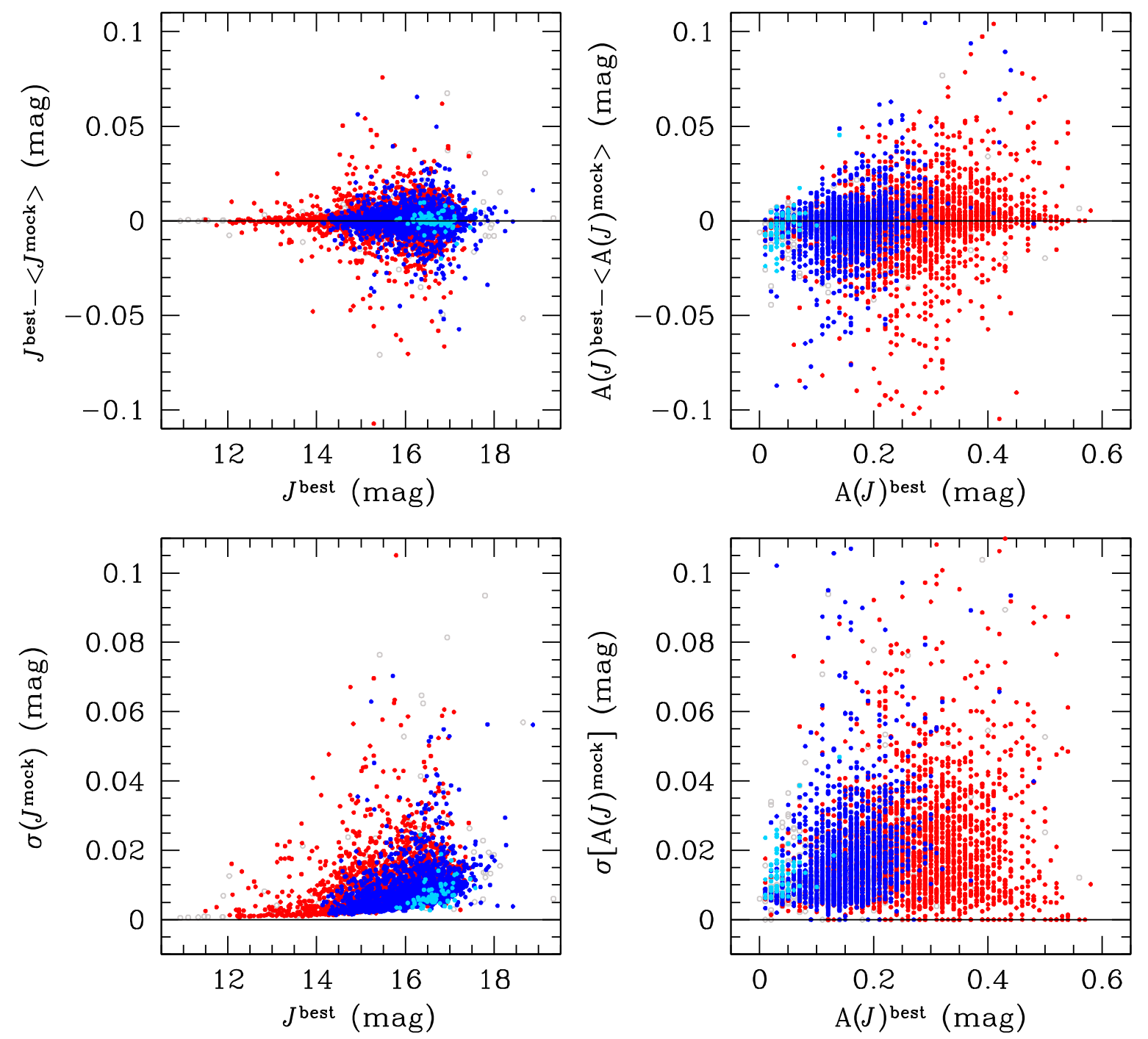

Figure 8. As Figure 7 but for the $J$ band.

Ripepi et al. 2012b), and which is thus particularly appropriate for our purpose. As a result, we found that there is a clear change in slope at $\log P=0.47$ ( 2.95 days) for F-mode pulsators, while there is no significant change in slope for FO variables. This was confirmed by the analysis of the PL and PW in different filters and is in agreement with the results obtained in the optical ( $V, I$ bands) for the OGLE III sample of F-mode pulsators by Subramanian \& Subramaniam and references therein. However, we do not find the break at $\log P=0.029(\sim 1.07$ days $)$ that they noticed for FO-mode pulsators. A possible explanation for the break detected at $P \sim 3$ days is that for shorter periods the blue loop of the Cepheid evolutionary track is too short and enters only the reddest part of the instability strip (M. Marconi et al. in prep).

The PW $\left(V, K_{\mathrm{s}}\right)$ was also used to analyze problematic objects, identified as clear outliers from these relations. In total, we discarded 223 CCs. We identified different (but often concurrent) reasons for the erratic behavior of these objects (see the final column in Table 5 for details): (i) misidentification: all objects with separation VMC-OGLE III > 0 ". 2 were visually inspected and rejected if they were found to be overluminous in the $\mathrm{PW}\left(V, K_{\mathrm{s}}\right)$ relation (more than 100 objects were rejected as such); (ii) scattered or heavily undersampled light curves (always low $G /$ high $\chi^{2}$ values; more than 50 such objects were present); (iii) notes from either OGLE III or VMC, i.e., the presence of flags reporting problems with the images (more than 20 rejections); (iv) saturation (seven objects). Note also that 17 objects with good VMC photometry were rejected because they lacked OGLE III $V$-band photometry. Not all outliers can be explained by invoking these reasons; in fact, there are 35 outliers for which we could find no apparent flaws. However, most are faint and all are overluminous. Hence, it is likely that they suffer from blending with bright neighbor stars. The discarded stars are reported separately, both in Table 5 and Figures 10-12. Finally, we note that a few other objects were excluded from the derivation of the PL, PW, and PLC relations involving the $Y$ or $J$ bands because of specific problems in these bands. To avoid confusion, these objects have not been highlighted in the table and figures.

On this basis, we performed a least-squares fit to the data to derive all relations, adopting a break at $\log P=0.47$ $(P \sim 2.95$ days) for F-mode CCs, while all FO- and SO-mode pulsators were used together. The results of this work are shown in Table 6 and Figures 17-19, where from top to bottom we display the F-, FO-, and SO-mode PL, PW, and PLC relations, respectively. Note that the PLC relations show a 

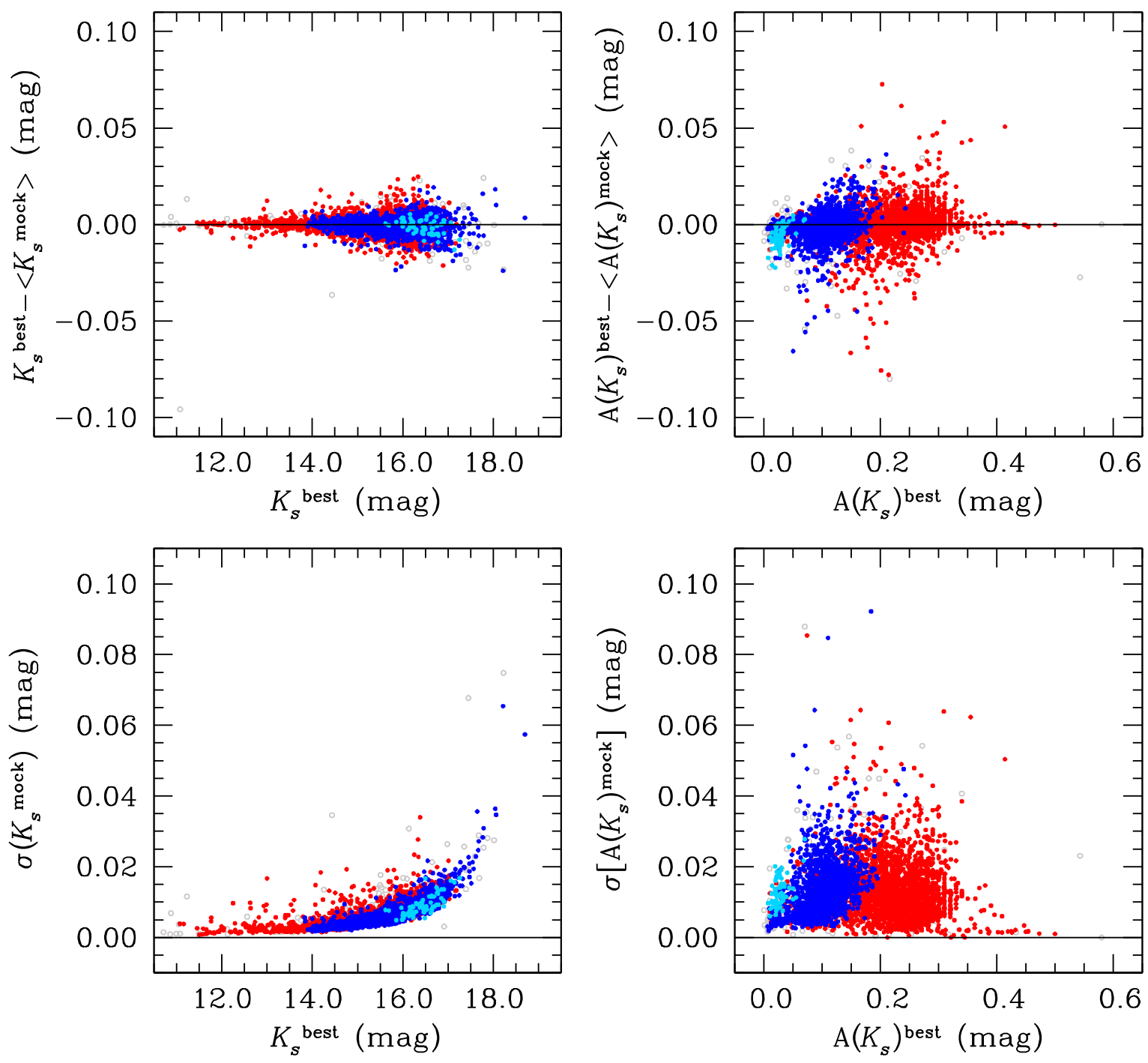

Figure 9. As Figure 7 but for the $K_{\mathrm{s}}$ band.

distinct discontinuity at $\log P=0.47$ owing to the way the data are projected in two dimensions. As far as we know, these are the first CC PL, PW, and PLC relations ever derived that involve the $Y$ band. The same is true for SO pulsators, even if in this case the small number of objects available for the calculations (about 70) and their intrinsic faintness did not allow us to obtain PL, PW, and PLC relations to similar precision as those for $\mathrm{F}$ and FO pulsators (see Table 6).

The regressions listed in Table 6 (and in Figures 17-19) show that the PL relations have, as expected, a larger dispersion with respect to the PW and PLC relations, which show a similar scatter for all combinations of magnitudes and colors, even though the use of $\left(V-K_{\mathrm{s}}\right)$ and $\left(J-K_{\mathrm{s}}\right)$ give slightly better results. This is not surprising, since the general quality of the $Y$ band data is (moderately) worse than that in $J$. In any case, the constancy of the dispersion of all these relations is a clear indication that the elongated structure of the SMC is dominating the intrinsic dispersion of these relations, which we know from LMC studies to be much smaller (see, e.g., Ripepi et al. 2012b; Inno et al. 2013; Macri et al. 2015).

We can now compare our results with the previous investigation by Inno et al. (2013). These authors derived PW relations for a variety of combinations of magnitudes and colors for SMC and LMC CCs, including the NIR bands $J, H, K_{\mathrm{s}}$. Their photometric database relies mainly on single-epoch light curves, from which they derived average magnitudes by adopting some literature template light curves and relying on published ephemerides and amplitude ratios (e.g., $A(J) / A(I)$ ). It is important to note our very different approach with respect to theirs. Indeed, the larger number of observed epochs (especially in the $K_{\mathrm{s}}$ band) allowed us to adopt a template procedure without having to rely on any external information (apart from the periods, see details in Section 3) and which is capable of achieving much higher precision of the intensity-averaged photometry for each individual CC. The relations we can compare with Inno et al. (2013) are the $\mathrm{PW}\left(J, K_{\mathrm{s}}\right)$ and $\mathrm{PW}\left(V, K_{\mathrm{s}}\right)$ for $\mathrm{F}$ and FO pulsators. The latter authors calculated these relations in different ways, either without taking into account any break or by arbitrarily imposing breaks at $\log P=0.35,0.40,0.45$. Therefore, we can compare the $\mathrm{PW}\left(J, K_{\mathrm{s}}\right)$ and $\mathrm{PW}\left(V, K_{\mathrm{s}}\right)$ relations with no break for FO pulsators (Table 1 of Inno et al. 2013) and the $\mathrm{PW}\left(J, K_{\mathrm{s}}\right)$ relation for $\mathrm{F}$ pulsators with a break at $\log P \geqslant 0.45$ (see Table 3 of Inno et al. 2013). ${ }^{20}$ To take into account that our photometry is in the

\footnotetext{
$\overline{20}$ Note that Inno et al. (2013)'s $\mathrm{PW}\left(V, K_{\mathrm{s}}\right)$ relations are not provided for different breaks, nor do they have relations for $\log P>0.45$.
} 

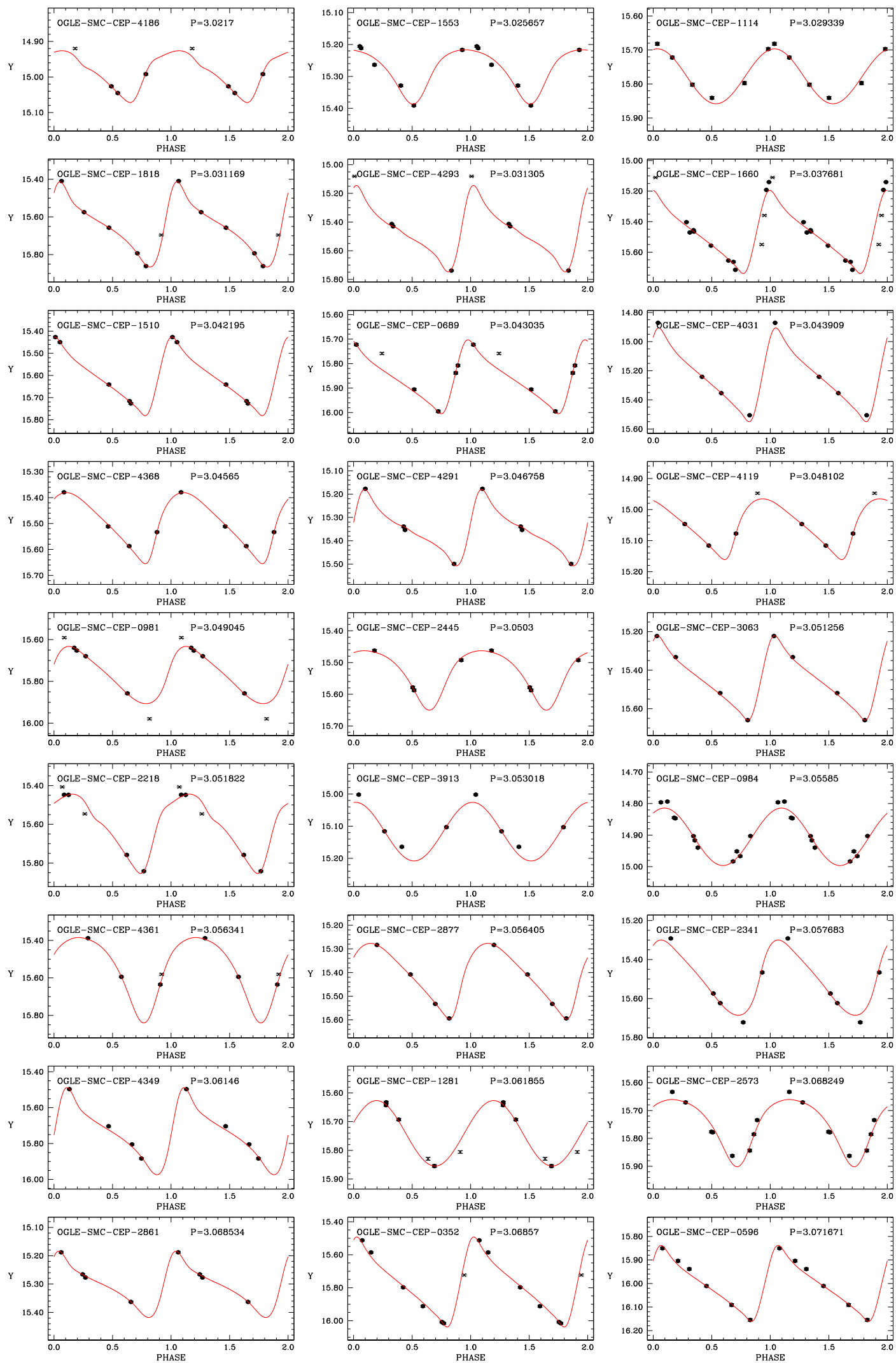

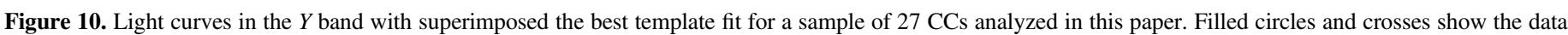

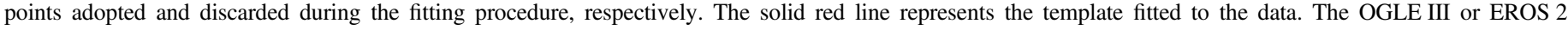

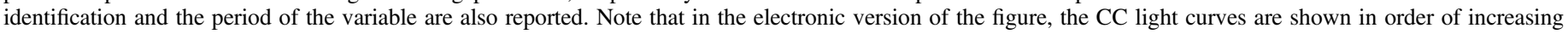
period (see caption of Table 5 for details).

(The complete figure set (156 images) is available.) 

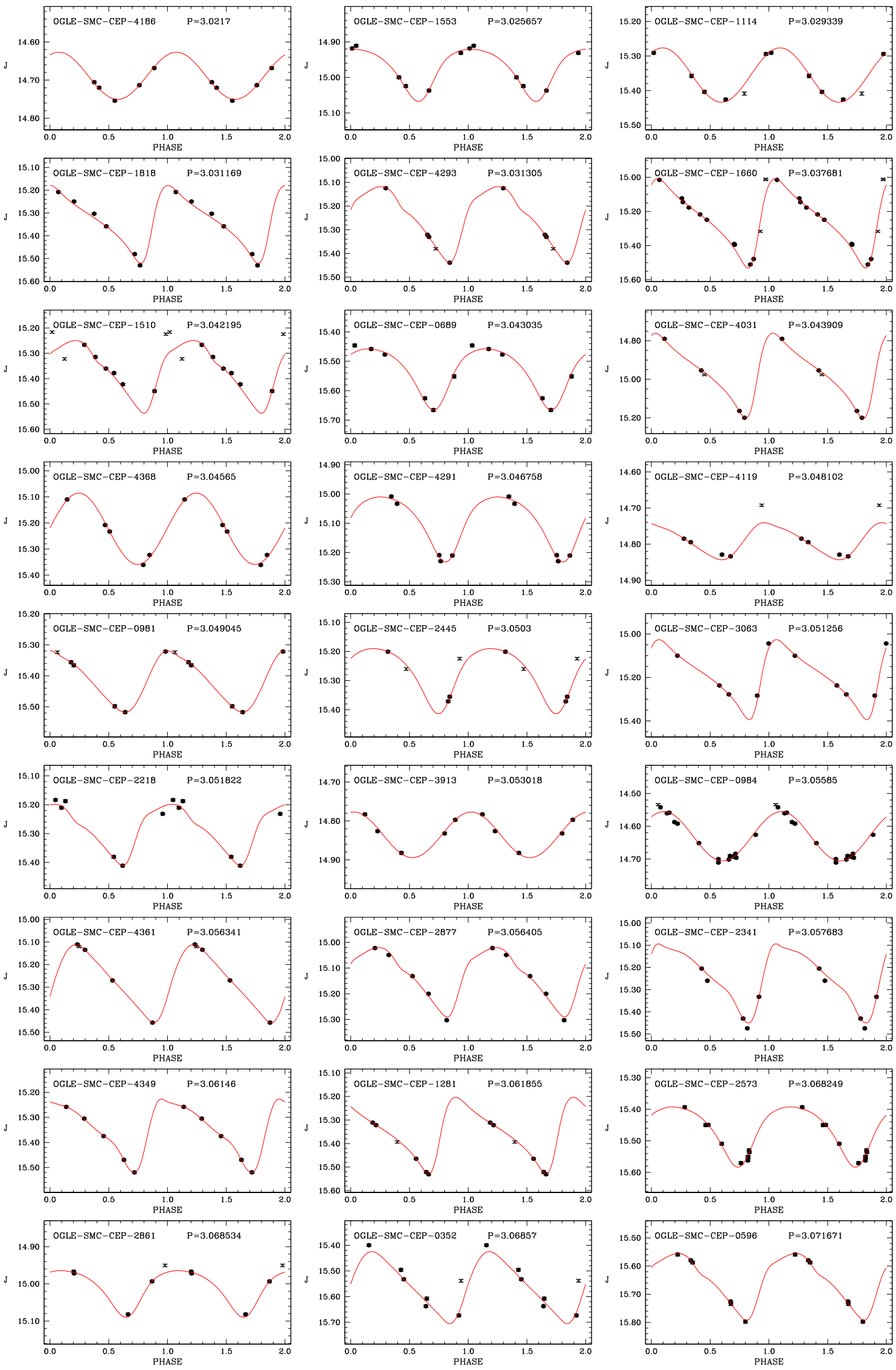

Figure 11. As Figure 10 but for the $J$ band.

(The complete figure set (156 images) is available.) 

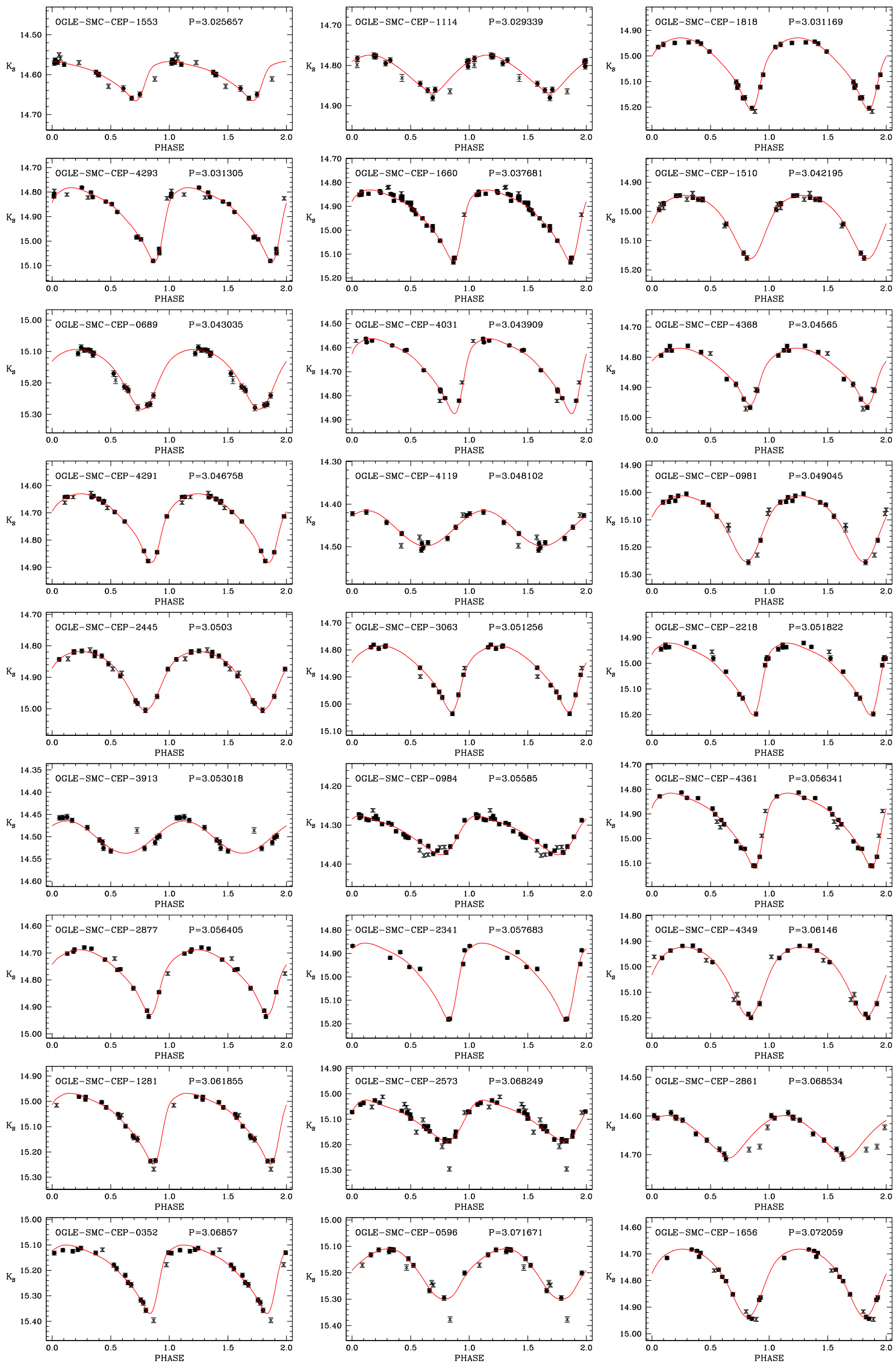

Figure 12. As Figure 10 but for the $K_{\mathrm{s}}$ band.

(The complete figure set (156 images) is available.) 


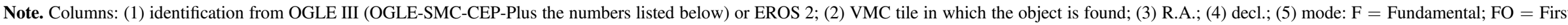

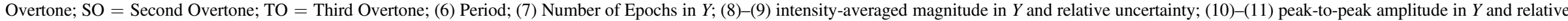

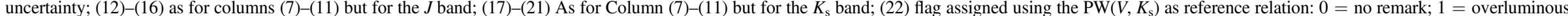

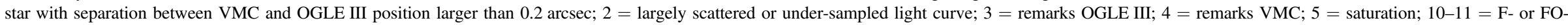

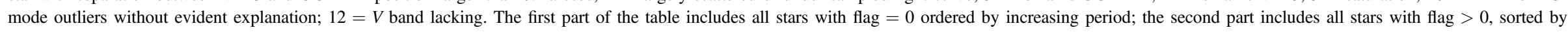
increasing period. The sorting of the table is the same as in Figures 10-12. We show the first 20 rows of the table to indicate its form and content.

(This table is available in its entirety in machine-readable form.) 

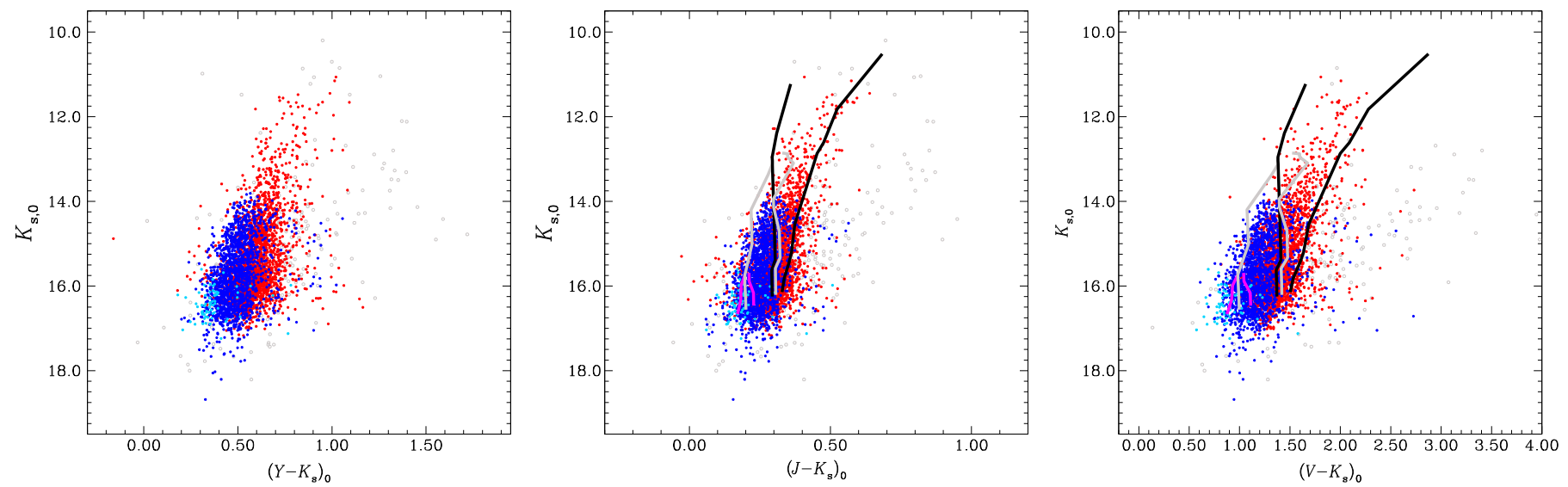

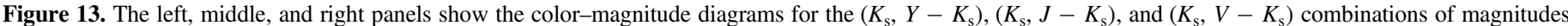

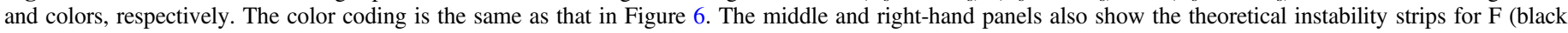

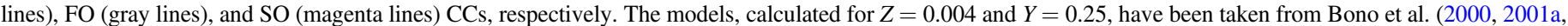
2001b).

VISTA system, while Inno et al. (2013)'s were in the 2MASS system, we have applied the equations discussed in Section 3 to convert Inno et al. (2013)'s relations to the VISTA system. We can now finally perform the comparison with the values listed in our Table 6. We obtain very good agreement for the three PW relations quoted above, in all cases within $\sim 1 \sigma$. However, we emphasize that the precision for the individual $\mathrm{CC} W$ magnitudes is better in our case given the larger number of observations. This is an important factor when dealing with the structure of the SMC, whose study requires precise individual relative distances.

\subsection{The Relative Distance between SMC and LMC and the Absolute Distance of the SMC}

The relationships derived in the previous Section will be used in a forthcoming paper to study in detail the 3D structure of the SMC. However, a first important use of the data presented in this paper is the estimation of the relative distance between the two MCs. In turn, the assumption of a distance for the LMC, which can be more safely determined with respect to the SMC's (since the SMC is so significantly elongated), allows us to provide an estimate of the absolute distance to the SMC (or, rather, of the center defined by the CC distribution).

We hence proceeded using our own data published in Ripepi et al. (2012b) for the CCs in the LMC. This is justified because (i) we used data in the same photometric system, (ii) we obtained a $\mathrm{PW}\left(V, K_{\mathrm{s}}\right)$ relation with very low dispersion for the LMC CCs, and (iii) we also provided an absolute distance estimate for the LMC.

The technique adopted is illustrated in Figure 20, where we compare the $\mathrm{PW}\left(V, K_{\mathrm{s}}\right)$ relations for $\mathrm{F}$ - and $\mathrm{FO}$-mode pulsators (top and bottom panels, respectively). First, observing the period distribution of the $\mathrm{CCs}$ in the LMC and the fact that the slope of the LMC's $\mathrm{PW}\left(V, K_{\mathrm{s}}\right)$ relation is very close to the slope we have found here for the SMC CCs characterized by $\log P>0.47$, we used this latter relation for our comparison of F-mode pulsators. Nonetheless, the slopes of the relations for both F- and FO-mode pulsators are slightly different for the LMC and SMC (which is possibly related to a weak but significant metallicity dependence). Indeed, it is possible to describe the difference in $W\left(V, K_{\mathrm{S}}\right)$, which translates directly into a difference in distance modulus $\mu$ as a function of period with two simple equations:

$$
\begin{aligned}
& \Delta \mu_{\mathrm{F}}=(0.49 \pm 0.02)+(0.06 \pm 0.03) \log P \\
& \Delta \mu_{\mathrm{FO}}=(0.58 \pm 0.01)-(0.06 \pm 0.03) \log P
\end{aligned}
$$

where $\Delta \mu$ means the difference in distance modulus of SMC and $\mathrm{LMC}$, and the errors take into account the uncertainties in both the LMC and SMC relations. To use Equations (10) and (11), we have to fix pivoting periods to determine the $\Delta \mu$ values. After some tests we chose $P=10$ days and $P=2$ days for F- and FO-mode pulsators, respectively. These values are approximately in the middle of the period range for both pulsator types, but it is easy to verify that the results do not depend significantly on this choice. The result of this exercise gives: $\Delta \mu_{\mathrm{F}}=0.55 \pm 0.04 \mathrm{mag} \quad$ and $\Delta \mu_{\mathrm{FO}}=0.56 \pm$ $0.03 \mathrm{mag}$, in excellent mutual agreement. Averaging the two results, we obtain our best estimate for the relative distance between the MCs: $\Delta \mu=0.55 \pm 0.04 \mathrm{mag}$. This value is in good agreement with that derived in a similar fashion by Inno et al. (2013), especially with their result for FO pulsators: $\Delta \mu=0.52 \pm 0.03 \mathrm{mag}$, while for F-mode CCs they find $\Delta \mu=0.48 \pm 0.03 \mathrm{mag}$. Our estimate is somewhat larger than those quotes in other papers based on different standard candles (see de Grijs et al. 2014, for a large compilation of distance differentials). For example, Cioni et al. (2000b) found $\Delta \mu=0.44 \pm 0.05$ from the tip of the red-giant branch, while using RR Lyrae stars Szewczyk et al. (2009) found a significantly smaller value, $\Delta \mu=0.327 \pm 0.002$ mag. According to Matsunaga et al. (2011), Type II Cepheids (W Vir) yield $\Delta \mu=0.40 \pm 0.07$ or $\Delta \mu=0.39 \pm 0.05 \mathrm{mag}$ (depending on the use of NIR or optical data, respectively). In general, Table 4 of Matsunaga et al. (2011), where they list several literature results, seems to suggest that all evaluations of the $\Delta \mu$ based on CCs provide larger values with respect to those based on population II indicators. This can be due to the very different spatial distribution among CCs (typically showing a disk-like location in both MCs) and population II tracers (e.g., RR Lyrae stars, type II Cepheids, which are more evenly distributed around a sort of spheroid in both MCs), as 

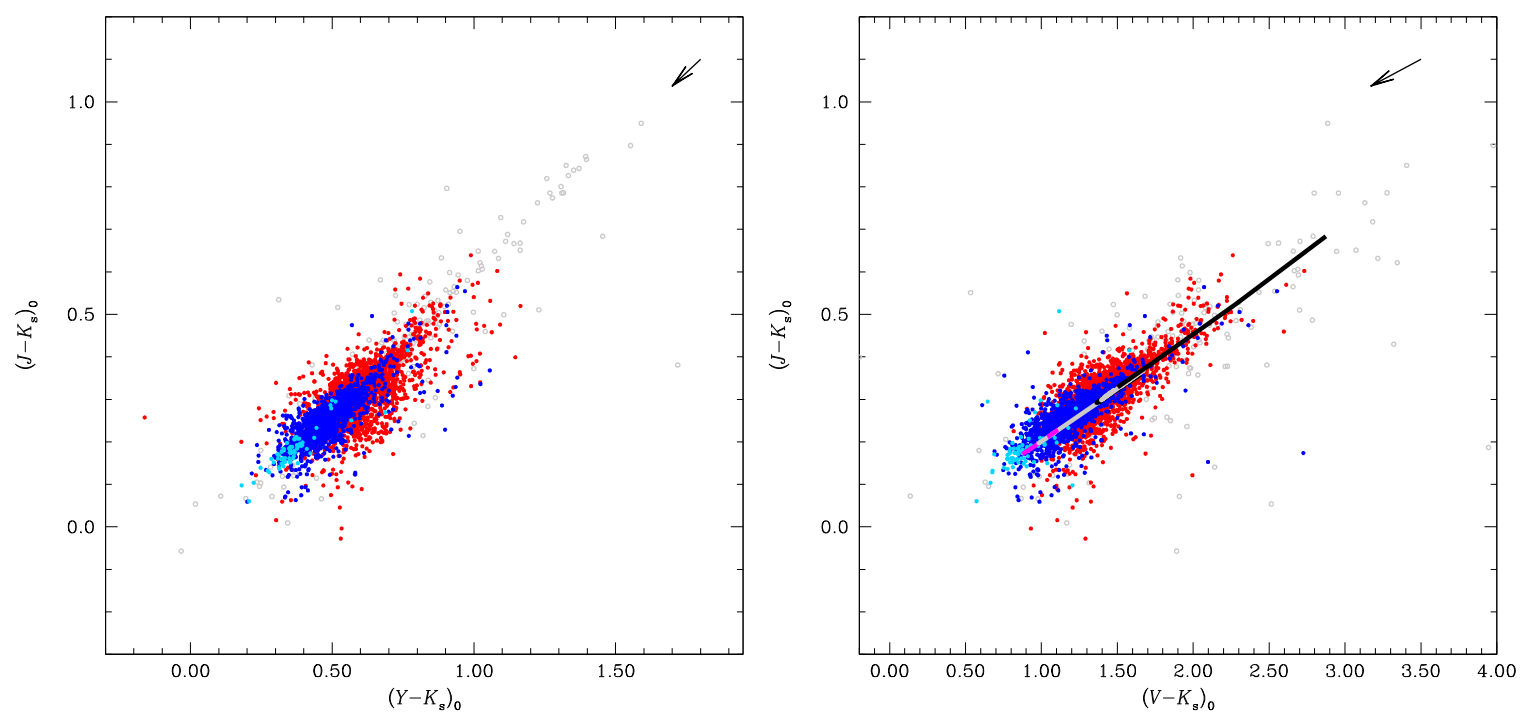

Figure 14. The left- and right-hand panels show the color-color diagrams in the $\left(J-K_{\mathrm{s}}, Y-K_{\mathrm{s}}\right)$ and $\left(V-K_{\mathrm{s}}, J-K_{\mathrm{s}}\right)$ planes, respectively. The color coding is the same as that in Figure 6. The right-hand panel also shows the theoretical instability strips from Figure 13. In both panels, arrows indicate the reddening vectors, calculated for a reddening value $E(V-I)=0.15 \mathrm{mag}$, i.e., approximately three times the average reddening in the SMC, according to the adopted reddening maps of Haschke et al. (2011).

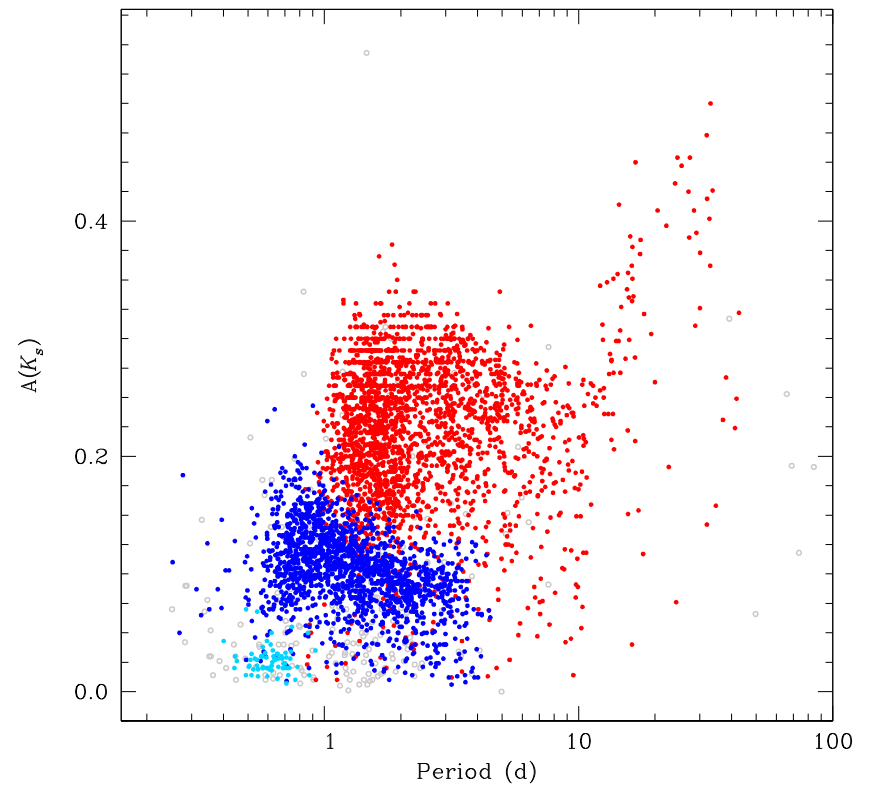

Figure 15. Period vs. amplitude in the $K_{\mathrm{s}}$ band for the target CCs. The color coding is the same as that in Figure 6.

shown, e.g., by Deb \& Singh (2014), Moretti et al. (2014), and Deb et al. (2015).

The absolute distance to the SMC can be determined by simply adding to the $\Delta \mu$ estimated above the preferred absolute distance for the LMC. There are hundreds of such estimates in the literature (see de Grijs et al. 2014, for a thorough review), but here we will consider in particular two values: (1) $\mu_{\text {LMC }}=18.46 \pm 0.03$ mag obtained in our previous work on LMC CCs (Ripepi et al. 2012b), and (2) $\mu_{\text {LMC }}=18.49 \pm 0.05$, accurately estimated by Pietrzyński et al. (2013) on the basis of an eclipsing Cepheid binary star. As a result, we obtain (1) $\mu_{\mathrm{SMC}}=19.01 \pm 0.05 \mathrm{mag}$ and (2) $\mu_{\mathrm{SMC}}=19.04 \pm 0.06 \mathrm{mag}$. These values are formally in agreement within $\sim 1 \sigma$ with that obtained in de Grijs \& Bono (2015) by averaging a large number of literature estimates:

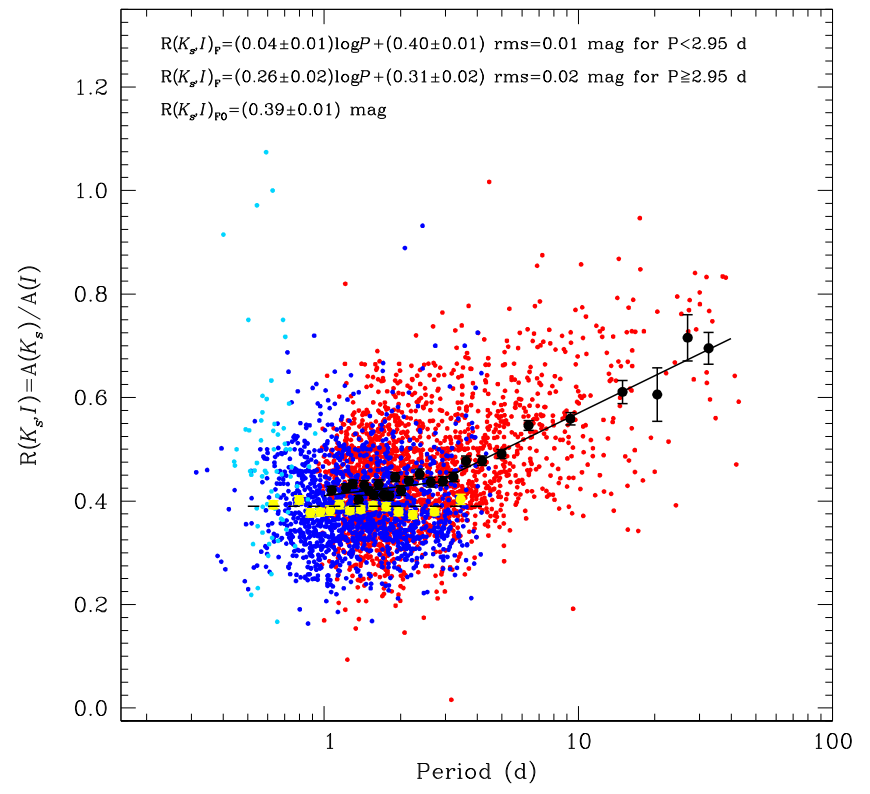

Figure 16. Peak-to-peak amplitude ratios $A\left(K_{\mathrm{s}}\right) / A(I)$ for the F, FO, and SO mode pulsators studied here. The $I$-band amplitudes band are from the OGLE III survey. The color coding of the small filled circles is as that in Figure 6. The black and yellow filled circles represent the averages in period bins for $\mathrm{F}$ and FO pulsators, respectively. The derived analytical ratios are labelled in the figure.

$\mu_{\mathrm{SMC}}=18.96 \pm 0.02 \mathrm{mag}$. However, as noted by these latter authors, the systematic uncertainty on this determination, caused by different sources (mainly the complex SMC geometry and its elongation along the line of sight), can be as large as $0.15-0.20 \mathrm{mag}$.

\section{CONCLUSIONS}

In this paper, we have presented the VMC survey's light curves for $4172 \mathrm{CCs}$ in the SMC. The majority of the objects have optical $V, I$ data as well as identification and periods from the OGLE III survey, while 13 CCs have been identified by the 
Table 6

PL, PW, and PLC Relations for F and FO CCs

\begin{tabular}{|c|c|c|c|c|c|c|c|}
\hline Mode & $\alpha$ & $\sigma_{\alpha}$ & $\beta$ & $\sigma_{\beta}$ & $\gamma$ & $\sigma_{\gamma}$ & $\mathrm{rms}$ \\
\hline \multicolumn{8}{|c|}{$Y^{0}=\alpha+\beta \log P$} \\
\hline $\mathrm{F} \log P<0.47$ & 17.247 & 0.011 & -3.413 & 0.043 & $\cdots$ & $\cdots$ & 0.196 \\
\hline $\mathrm{F} \log P \geqslant 0.47$ & 17.016 & 0.024 & -2.984 & 0.030 & $\cdots$ & $\cdots$ & 0.197 \\
\hline $\mathrm{FO}$ & 16.605 & 0.006 & -3.365 & 0.025 & $\cdots$ & $\cdots$ & 0.202 \\
\hline $\mathrm{SO}$ & 15.91 & 0.06 & -4.06 & 0.27 & $\cdots$ & $\cdots$ & 0.15 \\
\hline \multicolumn{8}{|c|}{$J^{0}=\alpha+\beta \log P$} \\
\hline $\mathrm{F} \log P<0.47$ & 16.978 & 0.010 & -3.469 & 0.040 & $\cdots$ & $\cdots$ & 0.182 \\
\hline $\mathrm{F} \log P \geqslant 0.47$ & 16.763 & 0.021 & -3.047 & 0.027 & $\cdots$ & $\cdots$ & 0.177 \\
\hline $\mathrm{FO}$ & 16.372 & 0.005 & -3.416 & 0.023 & $\cdots$ & $\cdots$ & 0.185 \\
\hline SO & 15.73 & 0.06 & -4.07 & 0.26 & $\cdots$ & $\cdots$ & 0.15 \\
\hline \multicolumn{8}{|c|}{$K_{\mathrm{s}}^{0}=\alpha+\beta \log P$} \\
\hline $\mathrm{F} \log P<0.47$ & 16.711 & 0.009 & -3.578 & 0.036 & $\cdots$ & $\cdots$ & 0.166 \\
\hline $\mathrm{F} \log P \geqslant 0.47$ & 16.513 & 0.019 & -3.195 & 0.024 & $\cdots$ & $\cdots$ & 0.156 \\
\hline $\mathrm{FO}$ & 16.133 & 0.005 & -3.544 & 0.020 & $\cdots$ & $\cdots$ & 0.169 \\
\hline $\mathrm{SO}$ & 15.52 & 0.06 & -4.28 & 0.26 & $\cdots$ & $\cdots$ & 0.15 \\
\hline \multicolumn{8}{|c|}{$W\left(Y, K_{\mathrm{s}}\right)=K_{\mathrm{s}}-0.42\left(Y-K_{\mathrm{s}}\right)=\alpha+\beta \log P$} \\
\hline $\mathrm{F} \log P<0.47$ & 16.489 & 0.009 & -3.660 & 0.035 & $\cdots$ & $\cdots$ & 0.158 \\
\hline $\mathrm{F} \log P \geqslant 0.47$ & 16.301 & 0.017 & -3.283 & 0.022 & $\cdots$ & $\cdots$ & 0.145 \\
\hline $\mathrm{FO}$ & 15.933 & 0.005 & -3.614 & 0.020 & $\cdots$ & $\cdots$ & 0.161 \\
\hline $\mathrm{SO}$ & 15.37 & 0.06 & -4.29 & 0.26 & $\cdots$ & $\cdots$ & 0.14 \\
\hline \multicolumn{8}{|c|}{$W\left(J, K_{\mathrm{s}}\right)=K_{\mathrm{s}}-0.69\left(J-K_{\mathrm{s}}\right)=\alpha+\beta \log P$} \\
\hline $\mathrm{F} \log P<0.47$ & 16.535 & 0.009 & -3.685 & 0.034 & $\cdots$ & $\cdots$ & 0.153 \\
\hline $\mathrm{F} \log P \geqslant 0.47$ & 16.343 & 0.017 & -3.301 & 0.021 & $\cdots$ & $\cdots$ & 0.139 \\
\hline $\mathrm{FO}$ & 15.964 & 0.005 & -3.618 & 0.019 & $\cdots$ & $\cdots$ & 0.156 \\
\hline $\mathrm{SO}$ & 15.39 & 0.06 & -4.31 & 0.26 & $\cdots$ & $\cdots$ & 0.15 \\
\hline \multicolumn{8}{|c|}{$W\left(V, K_{\mathrm{s}}\right)=K_{\mathrm{s}}-0.13\left(V-K_{\mathrm{s}}\right)=\alpha+\beta \log P$} \\
\hline $\mathrm{F} \log P<0.47$ & 16.559 & 0.008 & -3.666 & 0.033 & $\cdots$ & $\cdots$ & 0.147 \\
\hline $\mathrm{F} \log P \geqslant 0.47$ & 16.360 & 0.016 & -3.265 & 0.021 & $\cdots$ & $\cdots$ & 0.137 \\
\hline $\mathrm{FO}$ & 15.984 & 0.004 & -3.591 & 0.019 & $\cdots$ & $\cdots$ & 0.154 \\
\hline SO & 15.40 & 0.06 & -4.28 & 0.26 & $\cdots$ & $\cdots$ & 0.15 \\
\hline \multicolumn{8}{|c|}{$K_{\mathrm{s}}^{0}=\alpha+\beta \log P+\gamma\left(Y-K_{\mathrm{s}}\right)_{0}$} \\
\hline $\mathrm{F} \log P<0.47$ & 16.619 & 0.020 & -3.608 & 0.036 & 0.17 & 0.03 & 0.164 \\
\hline $\mathrm{F} \log P \geqslant 0.47$ & 16.239 & 0.035 & -3.312 & 0.026 & 0.55 & 0.06 & 0.146 \\
\hline $\mathrm{FO}$ & 15.923 & 0.023 & -3.629 & 0.021 & 0.44 & 0.05 & 0.163 \\
\hline $\mathrm{SO}$ & 15.62 & 0.10 & -4.21 & 0.28 & -0.25 & 0.19 & 0.16 \\
\hline \multicolumn{8}{|c|}{$K_{\mathrm{s}}^{0}=\alpha+\beta \log P+\gamma\left(J-K_{\mathrm{s}}\right)_{0}$} \\
\hline $\mathrm{F} \log P<0.47$ & 16.535 & 0.022 & -3.649 & 0.036 & 0.66 & 0.07 & 0.161 \\
\hline $\mathrm{F} \log P \geqslant 0.47$ & 16.227 & 0.032 & -3.372 & 0.028 & 1.16 & 0.11 & 0.144 \\
\hline FO & 15.911 & 0.021 & -3.657 & 0.021 & 0.92 & 0.08 & 0.162 \\
\hline $\mathrm{SO}$ & 15.64 & 0.08 & -4.23 & 0.27 & -0.65 & 0.30 & 0.16 \\
\hline \multicolumn{8}{|c|}{$K_{\mathrm{s}}^{0}=\alpha+\beta \log P+\gamma\left(V-K_{\mathrm{s}}\right)_{0}$} \\
\hline $\mathrm{F} \log P<0.47$ & 16.164 & 0.031 & -3.776 & 0.033 & 0.445 & 0.024 & 0.145 \\
\hline $\mathrm{F} \log P \geqslant 0.47$ & 15.879 & 0.035 & -3.498 & 0.024 & 0.543 & 0.027 & 0.121 \\
\hline FO & 15.676 & 0.025 & -3.710 & 0.020 & 0.402 & 0.022 & 0.149 \\
\hline $\mathrm{SO}$ & 15.39 & 0.12 & -4.28 & 0.26 & 0.14 & 0.12 & 0.15 \\
\hline
\end{tabular}

Note. The Wesenheit functions are defined in the table.

EROS 2 survey. Our data set consists of $Y, J$, and $K_{\mathrm{s}}$ light curves with the number of epochs typically ranging from 4 to 12 in $Y$ and $J$, and 13 to 36 in $K_{\mathrm{s}}$. We used our best light curves in each filter to construct samples of eight templates covering the full variety of periods and light-curve shapes. These templates have been used to automatically perform least- 


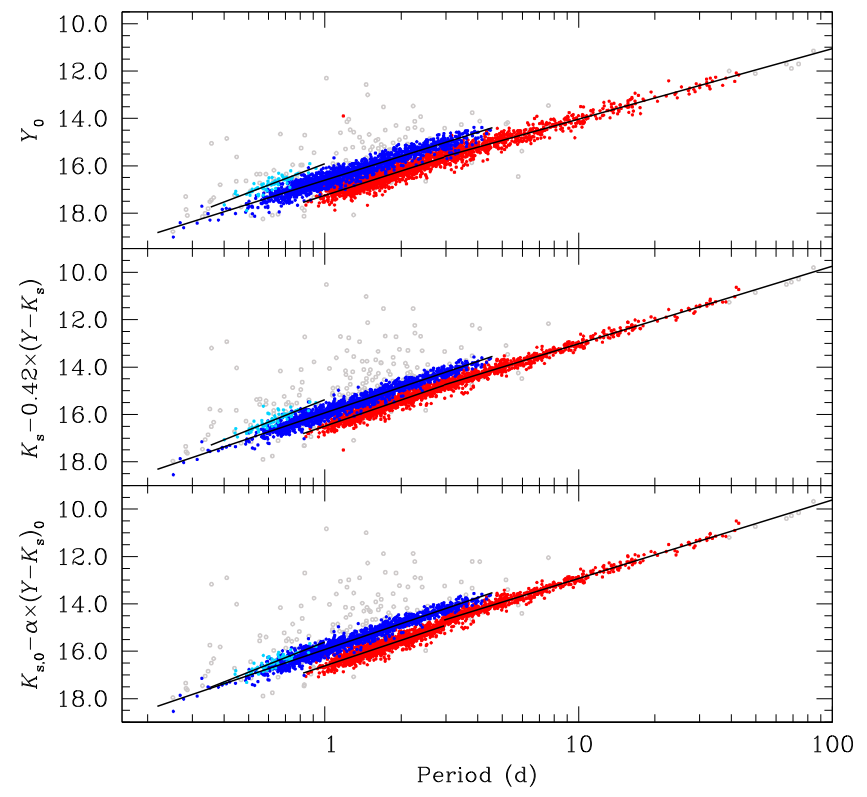

Figure 17. From top to bottom, $\operatorname{PL}(Y), \operatorname{PW}\left(K_{\mathrm{s}}, Y\right)$, and $\operatorname{PLC}\left(K_{\mathrm{s}}, Y\right)$ relations for the SMC CCs investigated in this paper. The color code is the same as that in Figure 6 . The solid lines represent least-squares fits to the data shown in Table 6. Note that the discontinuity in the bottom panel, both in data and fit, for F-mode pulsators is only due to the visualization procedure (projection from three- to two-dimensions).

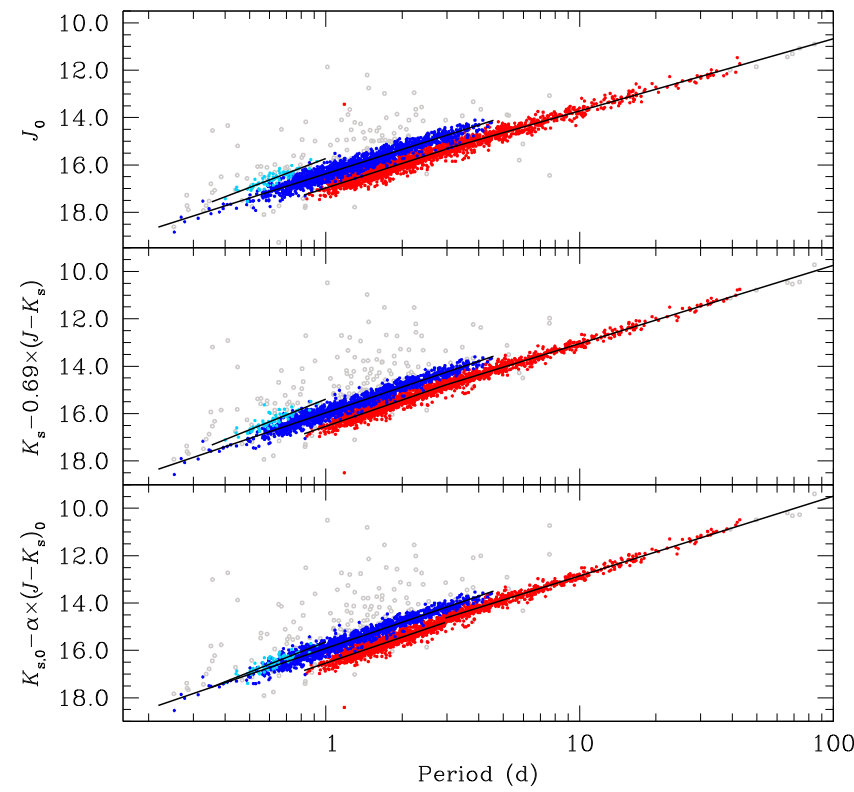

Figure 18. As Figure 17 but for $\operatorname{PL}(J), \operatorname{PW}\left(K_{\mathrm{s}}, J\right)$, and $\operatorname{PLC}\left(K_{\mathrm{s}}, J\right)$.

squares fits to the observations by varying both the amplitude and the phasing, and eventually choosing the best-fitting template by means of appropriately chosen parameters. We provide intensity-averaged magnitudes and peak-to-peak amplitudes in the $Y, J$, and $K_{\mathrm{s}}$ filters. To estimate reliable uncertainties on these values, we carried out Monte Carlo simulations, producing 100 mock light curves, adding Gaussian errors to the actual data for each $\mathrm{CC}$ in each filter, and running the template-fitting procedure from scratch each time. This process allowed us to assess the reliability of our templatefitting procedure and estimate robust uncertainties on $\mathrm{CC}$ magnitudes and amplitudes.

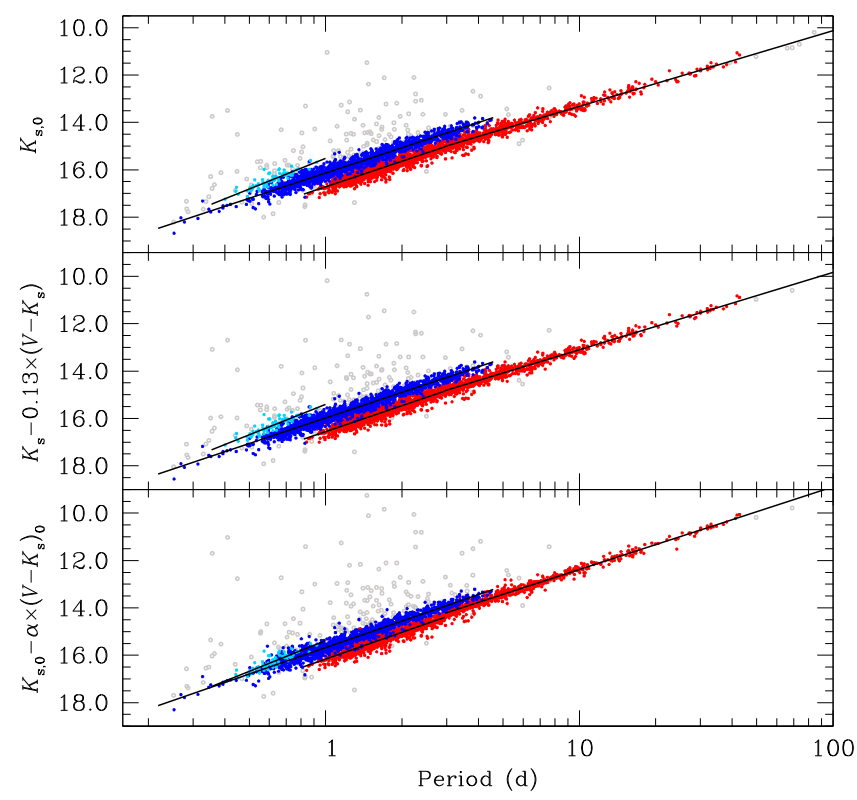

Figure 19. As Figure 17 but for $\operatorname{PL}\left(K_{\mathrm{s}}\right), \operatorname{PW}\left(K_{\mathrm{s}}, V\right)$, and $\operatorname{PLC}\left(K_{\mathrm{s}}, V\right)$.

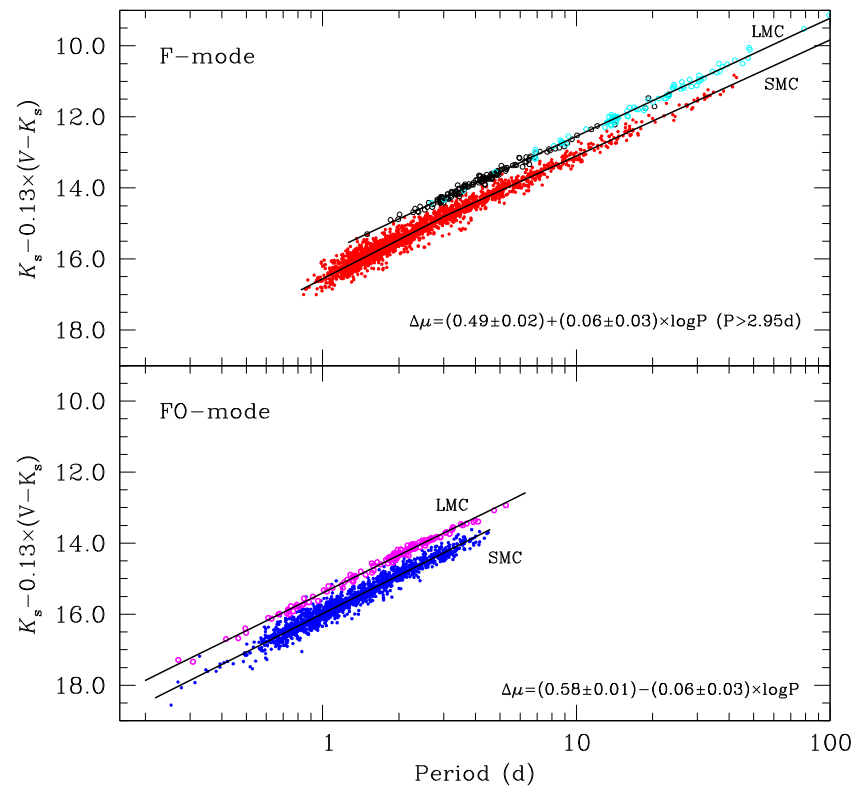

Figure 20. Top: $\mathrm{PW}\left(K_{\mathrm{s}}, V\right)$ for the $\mathrm{LMC}$ and SMC F-mode CCs according to Ripepi et al. (2012b, black circles), Persson et al. (2004, light blue circles), and this paper (filled red circles), respectively. Bottom: as the top panel but for FOmode pulsators in the LMC (magenta circles, Ripepi et al. 2012b) and in the SMC (filled blue circles, this work). In both panels the solid lines represent the best fits to the data (see text for details).

The intensity-averaged magnitudes in the VISTA $Y, J$, and $K_{\text {s }}$ filters have been complemented with optical $V$-band data and periods to construct a variety of PL, PW, and PLC relations for the CCs in the SMC. The relations involving $V, J$, and $K_{\mathrm{s}}$ are in agreement with those in the literature. As for the $Y$ band, to our knowledge in this paper we present the first CC PL, PW, and PLC ever obtained using this filter. The PL, PW, and PLC relations in the $V, J$, and $K_{\mathrm{s}}$ bands for F- and FO-mode CCs in the SMC presented here are the most accurate to date, since they are based on well- or moderately well-sampled light curves in $K_{\mathrm{s}}$ and $J$, respectively. We also presented the first NIR PL, PW, and PLC relations for SO pulsators to date. 
We used the $\mathrm{PW}\left(V, K_{\mathrm{s}}\right)$ relation to estimate the relative SMC-LMC distance and, in turn, the absolute distance of the SMC. For the former, we derive $\Delta \mu=0.55 \pm 0.04 \mathrm{mag}$, a value that is in rather good agreement with other evaluations based on CCs, but in disagreement (significantly larger) with estimates based on (old) population II distance indicators. We speculate that this discrepancy may be mainly due to the different geometric distribution of young and old tracers in the MCs.

As for the absolute distance to the SMC, our best estimates, $\mu_{\mathrm{SMC}}=19.01 \pm 0.05 \mathrm{mag}$ and $\mu_{\mathrm{SMC}}=19.04 \pm 0.06 \mathrm{mag}$, based on two particular evaluations of the distance to the LMC, are in good agreement with literature values. However, we have to take into account the large systematic uncertainty due to the complex geometry of the SMC. In a forthcoming paper, we will use our precise $\mathrm{PW}$ relations to unveil the $3 \mathrm{D}$ structure of the SMC. For the reasons outlined above, this work is also expected to reduce the systematic uncertainties associated with the absolute distance to the SMC.

We thank our anonymous referee for pertinent and helpful comments. This paper is based on observations collected at the European Organisation for Astronomical Research in the Southern Hemisphere under ESO programme(s) 179.B-2003. V.R. warmly thanks Roberto Molinaro for providing the software for the spline interpolation and the Fourier analysis used to construct the template light curves. Partial financial support for this work was provided by PRIN-INAF 2014 project "EXCALIBURS (PI: G. Clementini) and by PRINMIUR grant 2010LY5N2T_005 (PI: F. Matteucci). We thank the UKs VISTA Data Flow System comprising the VISTA pipeline at CASU and the VISTA Science Archive at Wide Field Astronomy Unit (Edinburgh; WFAU) for providing calibrated data products supported by the STFC. This work was partially supported by the Gaia Research for European Astronomy Training (GREAT-ITN) Marie Curie network, funded through the European Union Seventh Framework Programme ([FP7/2007-1312 2013] under grant agreement no. 264895). M.-R.L.C. acknowledges support from the German Exchange service. This work is supported by STFC grants ST/5001333 and ST/M001008. R.d.G. acknowledges financial support from the National Natural Research Foundation of China (grant 11373010).

\section{REFERENCES}

Bessell, M. S., \& Brett, J. M. 1988, PASP, 100, 1134

Bono, G., Caputo, F., Castellani, V., \& Marconi, M. 1999, ApJ, 512, 711

Bono, G., Caputo, F., \& Marconi, M. 2001a, MNRAS, 325, 1353

Bono, G., Caputo, F., Marconi, M., \& Musella, I. 2010, ApJ, 715, 277

Bono, G., Castellani, V., \& Marconi, M. 2000, ApJ, 529, 293

Bono, G., Gieren, W. P., Marconi, M., \& Fouqué, P. 2001b, ApJL, 552, L141

Caputo, F., Marconi, M., \& Musella, I. 2000, A\&A, 354, 610

Cardelli, J. A., Clayton, G. C., \& Mathis, J. S. 1989, ApJ, 345, 245

Carini, R., Brocato, E., Marconi, M., \& Raimondo, G. 2014, A\&A, 561, A110

Carpenter, J. M. 2001, AJ, 121, 2851

Cioni, M.-R. L., Clementini, G., Girardi, L., et al. 2011, A\&A, 527, A116

Cioni, M.-R. L., Habing, H. J., \& Israel, F. P. 2000a, A\&A, 358, L9

Cioni, M.-R. L., van der Marel, R. P., Loup, C., \& Habing, H. J. 2000b, A\&A, 359, 601

Cross, N. J. G., Collins, R. S., Mann, R. G., et al. 2012, A\&A, 548, A119
Dalton, G. B., Caldwell, M., Ward, A. K., et al. 2006, Proc. SPIE, 6269, $62690 X$

de Grijs, R., \& Bono, G. 2015, AJ, 149, 179

de Grijs, R., Wicker, J. E., \& Bono, G. 2014, AJ, 147, 122

Deb, S., \& Singh, H. P. 2014, MNRAS, 438, 2440

Deb, S., Singh, H. P., Kumar, S. \& Kanbur, S. M. 2015, MNRAS, 449, 2768

Dobbie, P. D., Cole, A. A., Subramaniam, A., \& Keller, S. 2014, MNRAS, 442,1663

Emerson, J., McPherson, A., \& Sutherland, W. 2006, Msngr, 126, 41

Emerson, J. P., Irwin, M. J., Lewis, J., et al. 2004, Proc. SPIE, 5493, 41

Fiorentino, G., Caputo, F., Marconi, M., \& Musella, I. 2002, ApJ, 576, 402

Fiorentino, G., Musella, I., \& Marconi, M. 2013, MNRAS, 434, 2866

Freedman, W. L. 1988, ApJ, 326, 691

Freedman, W. L., Madore, B. F., Gibson, B. K., et al. 2001, ApJ, 553, 47

Gao, J., Jiang, B. W., Li, A., \& Xue, M. Y. 2013, ApJ, 776, 7

Glatt, K., Grebel, E. K., Sabbi, E., et al. 2008, AJ, 136, 1703

Hammer, F., Yang, Y. B., Flores, H., Puech, M., \& Fouquet, S. 2015, ApJ, 813,110

Harris 2007, ApJ, 658, 345

Harris, J., \& Zaritsky, D. 2004, AJ, 127, 1531

Harris, J., \& Zaritsky, D. 2009, AJ, 138, 1243

Haschke, R., Grebel, E. K., \& Duffau, S. 2011, AJ, 141, 158

Haschke, R., Grebel, E. K., \& Duffau, S. 2012, AJ, 144, 107

Hatzidimitriou, D., \& Hawkins, M. R. S. 1989, MNRAS, 241, 667

Inno, L., Matsunaga, N., Bono, G., et al. 2013, ApJ, 764, 84

Inno, L., Matsunaga, N., Romaniello, M., et al. 2015, A\&A, 576, A30

Irwin, M. J. 2009, UKIRT Newsletter, 25, 15

Irwin, M. J., Kunkel, W. E., \& Demers, S. 1985, Natur, 318, 160

Irwin, M. J., Lewis, J., Hodgkin, S., et al. 2004, Proc. SPIE, 5493, 411

Kerber, L. O., Girardi, L., Rubele, S., \& Cioni, M.-R. 2009, A\&A, 499, 697

Kim, D.-W., Protopapas, P., Bailer-Jones, C. A. L., et al. 2014, A\&A, 566, A43

Macri, L. M., Ngeow, C.-C., Kanbur, S. M., Mahzooni, S., \& Smitka, M. T 2015, AJ, 149, 117

Madore, B. F. 1982, ApJ, 253, 575

Marconi, M. 2009, MmSAI, 80, 141

Marconi, M., Fiorentino, G., \& Caputo, F. 2004, A\&A, 417, 1101

Marconi, M., Musella, I., \& Fiorentino, G. 2005, ApJ, 632, 590

Marconi, M., Musella, I., Fiorentino, G., et al. 2010, ApJ, 713, 615

Matsunaga, N., Feast, M. W., \& Soszyński, I. 2011, MNRAS, 413, 223

Moretti, M. I., Clementini, G., Muraveva, T., et al. 2014, MNRAS, 437, 2702

Muraveva, T., Palmer, M., Clementini, G., et al. 2015, ApJ, 807, 127

Ngeow, C., Kanbur, S. M., \& Nanthakumar, A. 2008, A\&A, 477, 621

Nidever, D. L., Monachesi, A., Bell, E. F., et al. 2013, ApJ, 779, 145

Persson, S. E., Madore, B. F., Krzemiński, W., et al. 2004, AJ, 128, 2239

Pietrzyński, G., Graczyk, D., Gieren, W., et al. 2013, Natur, 495, 76

Putman, M. E., Gibson, B. K., Staveley-Smith, L., et al. 1998, Natur, 394, 752

Riess, A. G., Macri, L., Casertano, S., et al. 2011, ApJ, 730, 119

Ripepi, V., Cignoni, M., Tosi, M., et al. 2014a, MNRAS, 442, 1897

Ripepi, V., Marconi, M., Moretti, M. I., et al. 2014b, MNRAS, 437, 2307

Ripepi, V., Moretti, M. I., Clementini, G., et al. 2012a, Ap\&SS, 341, 51

Ripepi, V., Moretti, M. I., Marconi, M., et al. 2012b, MNRAS, 424, 1807

Ripepi, V., Moretti, M. I., Marconi, M., et al. 2015, MNRAS, 446, 3034

Romaniello, M., Primas, F., Mottini, M., et al. 2008, A\&A, 488, 731

Rubele, S., Girardi, L., Kerber, L., et al. 2015, MNRAS, 449, 639

Schaltenbrand, R., \& Tammann, G. A. 1971, A\&AS, 4, 265

Scowcroft, V., Freedman, W. L., Madore, B. F., et al. 2016, ApJ, 816, 49

Shapley, H. 1940, BHarO, 914, 8

Skrutskie, M. F., Cutri, R. M., Stiening, R., et al. 2006, AJ, 131, 1163

Soszyński, I., Gieren, W., \& Pietrzyński, G. 2005, PASP, 117, 823

Soszyński, I., Poleski, R., Udalski, A., et al. 2010, AcA, 60, 17

Stanimirović, S., Staveley-Smith, L., \& Jones, P. A. 2004, ApJ, 604, 176

Subramanian, S., \& Subramaniam, A. 2015, A\&A, 573, A135

Szewczyk, O., Pietrzyński, G., Gieren, W., et al. 2009, AJ, 138, 1661

Tisserand, P., Le Guillou, L., Afonso, C., et al. 2007, A\&A, 469, 387

Udalski, A., Szymanski, M., Kubiak, M., et al. 1999, AcA, 49, 201

Welch, D. L., McLaren, R. A., Madore, B. F., \& McAlary, C. W. 1987, ApJ, 321,162

Westerlund, B. E. 1997, The Magellanic Clouds (Cambridge: Cambridge Univ. Press)

Zaritsky, D., Harris, J., Grebel, E. K., \& Thompson, I. B. 2000, ApJL, 534, L53 